\title{
A flexible and scalable scheme for mixing computed formation energies from different levels of theory
}

\author{
Ryan Kingsbury, ${ }^{1,2}$ Andrew S. Rosen, ${ }^{1,3}$ Ayush S. Gupta, ${ }^{1}$ Jason Munro, ${ }^{3}$ Shyue Ping \\ Ong, ${ }^{4,3}$ Anubhav Jain, ${ }^{2}$ Shyam Dwaraknath, ${ }^{3}$ Matthew Horton, ${ }^{3}$ and Kristin A. Persson ${ }^{1,5}, *$ \\ ${ }^{1}$ Department of Materials Science and Engineering, \\ University of California Berkeley, Berkeley, CA 94720 \\ ${ }^{2}$ Energy Technologies Area \\ Lawrence Berkeley National Laboratory, Berkeley, CA 94720 \\ ${ }^{3}$ Materials Science Division \\ Lawrence Berkeley National Laboratory, Berkeley, CA 94720 \\ ${ }^{4}$ University of California San Diego, La Jolla, CA 92093 \\ ${ }^{5}$ Molecular Foundry \\ Lawrence Berkeley National Laboratory, Berkeley, CA 94720
}

(Dated: March 3, 2022)

\begin{abstract}
Phase stability predictions are central to computational materials discovery efforts and have been made possible by large databases of computed properties from high-throughput density functional theory (DFT) calculations. Such databases now contain millions of calculations at the generalized gradient approximation (GGA) level of theory, representing an enormous investment of computational resources. Although it is now feasible to carry out large numbers of calculations using more accurate methods, such as meta-GGA functionals, recomputing the entirety of a database with a higher-fidelity method is impractical and would not effectively leverage the value embodied in existing calculations. Instead, we propose in this work a general procedure by which higher-fidelity, low-coverage calculations (e.g., meta-GGA calculations for selected chemical systems) can be combined with lower-fidelity, high-coverage calculations (e.g., an existing database of GGA calculations) in a robust and scalable manner to yield improved phase stability predictions. We demonstrate our scheme using legacy GGA $(+U)$ calculations and new $\mathrm{r}^{2}$ SCAN meta-GGA calculations from the Materials Project and illustrate its application to solid and aqueous phase stability. We discuss practical considerations for constructing mixed phase diagrams and present guidelines for prioritizing high-fidelity calculations for maximum benefit.
\end{abstract}

\section{INTRODUCTION}

The advent of large databases of computed material properties, such as the Materials Project [1], AFLOW [2], the Open Quantum Materials Database (OQMD) [3, 4], and the Joint Automated Repository for Various Integrated Simulations (JARVIS) [5], has paved the way for a new era of data-driven materials science [6]. These databases now contain computed properties derived from millions of individual calculations, the vast majority of which employ density functional theory (DFT) due to its efficient compromise between computational cost and accuracy. For example, the Materials Project [1] contains computed formation energies for more than 140,000 materials calculated using the Perdew-Burke-Ernzerhof (PBE) [7] generalized gradient approximation (GGA) [8] functional, with a Hubbard $U$ value [9] and empirical energy corrections [10] applied to some chemical systems. This data is widely used in machine learning and computational materials screening efforts in which the thermodynamic (meta)stablility of a material is often among the first selection criteria [11].

Despite its versatility and historical success, PBE has well-documented systematic errors related to electron

* kapersson@lbl.gov; https://materialsproject.org/ self-interaction $[12,13]$ that are particularly notable in diatomic gases $[14,15]$ and transition metal compounds with localized electronic states $[1,15,16]$. PBE also fails to capture medium- and long-range dispersion interactions [17], which are important for describing the properties of weakly-bound systems. Even when adjusted using empirical correction schemes, the mean absolute error (MAE) in formation energies predicted by this level of theory are still on the order of 50-200 $\mathrm{meV}$ /atom $[10,14,15,18-22]$, although the error in energy differences among polymorphs is typically lower (e.g. $25 \mathrm{meV} /$ atom) [23].

Today, more than a decade after most materials databases were established [6], theoretical advances and growth in computing power have made it feasible to compute large numbers of formation energies at higher levels of theory [18], which could substantially increase their accuracy. For example, we recently showed that the restored-regularized Strongly Constrained and Appropriately Normed ( $\left.\mathrm{r}^{2} \mathrm{SCAN}\right)$ meta-GGA functional [24] reduced the error in predicted formation energies of strongly-bound and weakly-bound materials by $50 \%$ and 15\%, respectively, compared to the PBEsol [25] GGA functional, while simultaneously exhibiting reliable convergence [18]. The original SCAN functional [26] on which it is based has also been shown to predict volumes, lattice constants, and ground-state structures of 
many materials more accurately than PBE [22, 27-31].

Carrying out enough higher-fidelity calculations to comprehensively cover technologically-relevant chemical spaces, as is required for the construction of compositional phase diagrams and the discovery of new structure-property relationships, could clearly benefit the many materials discovery efforts that depend on such data. However, replacing all of the existing lowerfidelity (GGA) calculations in large materials databases with higher-fidelity (e.g., meta-GGA) calculations would consume an enormous amount of energy and computing time, since SCAN and $\mathrm{r}^{2} \mathrm{SCAN}$, for example, have 4$5 \times$ the computational cost of PBE [18, 22, 32]. Even if resources were unlimited, there is likely to be little benefit in recomputing materials that are highly unstable (i.e., far from the convex energy hull), since predicting (meta)stability is of primary importance. Furthermore, meta-GGA calculations will not improve formation energy predictions to an equal extent for all materials. For example, SCAN has been shown to be slightly less accurate than $\mathrm{PBE}$ in predicting the formation energies of weakly-bound materials (e.g., intermetallics [22]), and $r^{2}$ SCAN improves the predictions for these materials to a much lesser extent than for strongly-bound materials [18].

Therefore, instead of recomputing materials en masse, higher-fidelity calculations should be targeted at the materials for which they are likely to improve the accuracy of the phase diagrams the most (e.g., strongly-bound materials, materials close to the energy convex hull). Adopting this strategy will economize future use of resources and preserve the massive investment embodied in existing lower-fidelity calculations. Notably, however, such an approach will require phase stability predictions to be based on a mixture of formation energies computed at different levels of theory. The most straightforward way to systematically improve upon GGA phase diagrams in a high-throughput manner, which we refer to here as "naive mixing", is simply to build each phase diagram using formation energies from lower level calculations, and then replace them with higher level calculations whenever they are available. However as we will show, constructing mixed phase diagrams this way can result in severe distortions to the shape of the convex energy hull and dramatically worsen phase stability predictions.

As an alternative to naive mixing, we propose in this work a scheme to construct phase diagrams that mix calculations from different density functionals comprising a lower-fidelity, higher coverage and a higher-fidelity, lower coverage set of calculations (here, $\operatorname{PBE}(+U)$ and $\mathrm{r}^{2} \mathrm{SCAN}$ ) with minimal risk of distortion. By defining the reference state at each point in compositional space as the ground state $\mathrm{PBE}(+U)$ structure, we build a framework in which energies from any two functionals can be mixed in a robust and scalable manner that preserves the shape of the convex hull. After presenting our mixing scheme, we assess how a transition from $\mathrm{PBE}(+U)$ to $\mathrm{r}^{2} \mathrm{SCAN}$ affects predicted polymorph stability and energy above hull by analyzing a set of approximately 33,900 $\mathrm{r}^{2} \mathrm{SCAN}$ calculations and discuss strategies for prioritizing $\mathrm{r}^{2} \mathrm{SCAN}$ calculations such that the mixed phase diagram most closely approximates the full $\mathrm{r}^{2} \mathrm{SCAN}$ phase diagram. We conclude by using our mixing scheme to analyze solid and aqueous phase stability in two example systems. The mixed phase diagrams presented in this work, along with the $33,000+$ new $\mathrm{r}^{2} \mathrm{SCAN}$ calculations, are made publicly available in the Materials Project database [1] to increase the accuracy of future computational material science efforts.

\section{THEORETICAL FRAMEWORK FOR MIXING FORMATION ENERGIES FROM DIFFERENT FUNCTIONALS}

\section{A. Mixing rules}

The computed energy of formation for a material, $\Delta H_{f}$, is defined with respect to the elements by $[9,19$, $21,33]$

$$
\Delta H_{f}^{298 \mathrm{~K}, \mathrm{DFT}} \approx E^{0 \mathrm{~K}, \mathrm{DFT}}-\sum_{\mathrm{el}} n_{\mathrm{el}} E_{\mathrm{el}}^{0 \mathrm{~K}, \mathrm{DFT}}
$$

where $E^{0 \mathrm{~K}, \mathrm{DFT}}$ is the total electronic energy computed from DFT at $0 \mathrm{~K}$, subscript 'el' represents each of the constituent elements in the material, and $n$ are stoichiometric coefficients. We note that $E$ can include empirical corrections and that this formulation assumes that differences in finite-temperature enthalpy between materials are negligible. Electronic energies $E$ are not intrinsically meaningful, and their energy scales differ substantially among functionals for the same material. However, the differences in electronic energy among materials and elements, and hence the value of $\Delta H_{f}$, define a consistent, physically-meaningful quantity that can be compared among different levels of theory.

In this manuscript, we consider mixing $\mathrm{GGA}(+U)$ and $\mathrm{r}^{2} \mathrm{SCAN}$ calculations; although the scheme we present here can be used to mix energies from any two functionals. Note that we use "GGA $(+U)$ " to refer to the mixture of empirically-corrected $\mathrm{PBE}$ and $\mathrm{PBE}+U$ calculations that currently populate the Materials Project Database. Specifically, the Materials Project uses PBE (i.e., GGA) for all materials except those containing Co, $\mathrm{Cr}, \mathrm{Fe}, \mathrm{Mn}, \mathrm{Mo}, \mathrm{Ni}, \mathrm{V}$ and $\mathrm{W}$, which are calculated with a Hubbard $U$ value [34]. These GGA and GGA $+U$ calculations are combined using the mixing scheme of Jain et al. [9] to yield a consistent set of formation energies. In this work, we use this combination of adjusted GGA and GGA $+U$ calculations as our high-coverage, low-fidelity set of calculations, while " $\mathrm{r}^{2} \mathrm{SCAN}$ " denotes unadjusted meta-GGA energies that comprise the lowcoverage, higher-fidelity calculations. Additional details regarding the computational methods for each calculation type are provided in Section V. 
Rule \#1

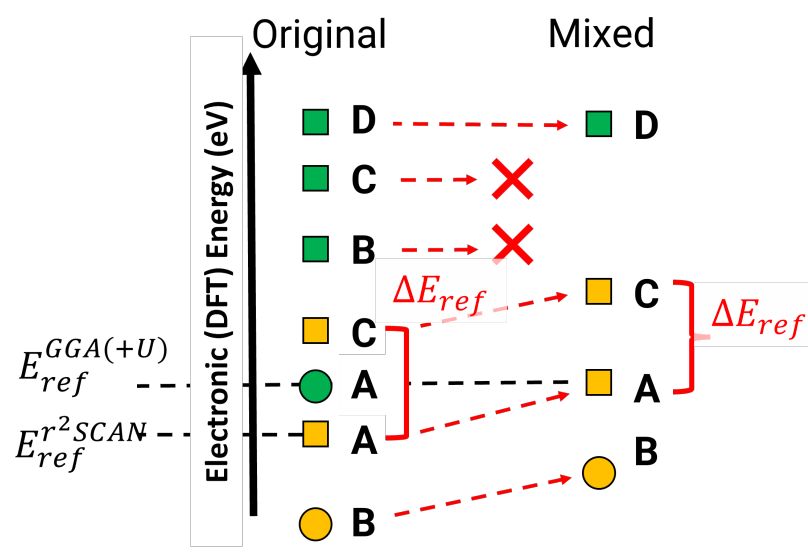

Rule \#2

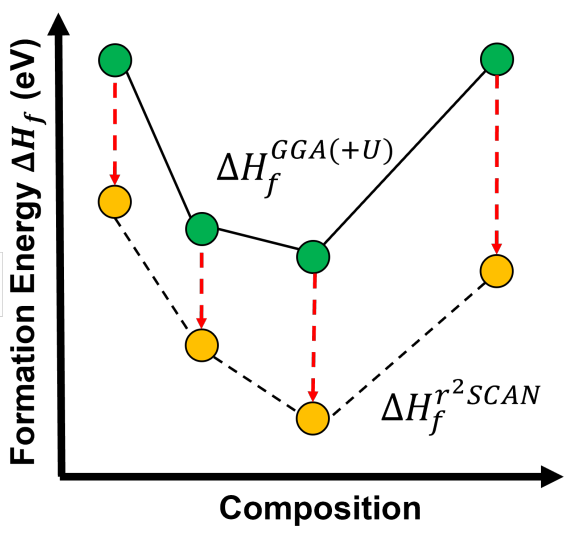

Composition

\section{GGA(+U) unstable polymorph $\square \mathrm{r}^{2}$ SCAN unstable polymorph $\mathrm{GGA}(+\mathrm{U})$ ground state}

FIG. 1. Rules for mixing GGA $(+U)$ (green) and $\mathrm{r}^{2} \mathrm{SCAN}$ (yellow) energies onto a single phase diagram. 1) $\mathrm{r}^{2} \mathrm{SCAN}$ energies can be placed onto a GGA $(+U)$ hull by referencing them to the $\mathrm{r}^{2} \mathrm{SCAN}$ energy of the GGA $(+U)$ ground state via $\Delta E_{r e f}$. In the diagram, A, B, C, and D represent different polymorphs at a single composition, and polymorph A is the GGA $(+U)$ ground state. $E_{r e f}^{G G A+U}$ and $E_{r e f}^{r^{2} S C A N}$ are the electronic (DFT) energies of polymorph A in the two functionals. GGA(+U) energies of polymorphs that also exist in $\mathrm{r}^{2} \mathrm{SCAN}$ (e.g., polymorphs B and C) are removed. 2) The convex hull is built with $\mathrm{r}^{2} \mathrm{SCAN}$ formation energies only when there are $\mathrm{r}^{2} \mathrm{SCAN}$ calculations for every $\mathrm{GGA}(+U)$ ground state.

As noted in the Introduction, the most straightforward approach to constructing mixed $\mathrm{r}^{2} \mathrm{SCAN} / \mathrm{GGA}(+U)$ phase diagrams is "naive mixing", where we simply replace GGA $(+U)$ formation energies with $\mathrm{r}^{2} \mathrm{SCAN}$ formation energies whenever $\mathrm{r}^{2} \mathrm{SCAN}$ calculations are available, while using $\mathrm{GGA}(+U)$ formation energies everywhere else. There are two drawbacks with naive mixing. First, in chemical systems where $\mathrm{r}^{2} \mathrm{SCAN}$ predicts significantly smaller or larger formation energies than $\mathrm{GGA}(+U)$ for most compounds, inserting a single $\mathrm{r}^{2} \mathrm{SCAN}$ formation energy onto a $\mathrm{GGA}(+U)$ phase diagram can either cause that single phase to move off the hull (if $\Delta H_{f}^{\mathrm{r}^{2} \mathrm{SCAN}} \gg \Delta H_{f}^{\mathrm{GGA}(+U)}$ ), or cause adjacent phases to disappear from the hull (if $\Delta H_{f}^{\mathrm{r}^{2} \mathrm{SCAN}} \ll \Delta H_{f}^{\mathrm{GGA}(+U)}$ ), when neither would occur on a full $\mathrm{r}^{2} \mathrm{SCAN}$ phase diagram. Second, in many cases $\mathrm{r}^{2} \mathrm{SCAN}$ stabilizes a different ground-state structure for the elements than GGA $(+U)$ (see Appendix B). Naive mixing of formation energies in chemical systems containing one of these elements is not rigorously consistent, because the formation energies are being referenced to different structures.

To circumvent these issues, we build our mixing scheme by considering all formation energies to be the sum of a reference energy and a relative energy. We define the reference energy, $E_{r e f}$, for each functional as the formation energy of the $G G A(+U)$ ground state structure at each point in compositional space, where the formation energy is calculated with respect to elemental end- points relaxed with that functional. The energy of any material in either functional may then be expressed as a difference, $\Delta E_{\text {ref }}$, relative to the corresponding reference energy. Note that $\Delta E_{\text {ref }}$ is calculated directly from the difference in polymorph energies, and hence does not depend on the energies of the elemental endpoints. Our mixing scheme is similar in spirit to the previous GGA/GGA $+U$ mixing scheme as well as the combined computational-experimental Pourbaix diagrams of the Materials Project [9, 35]; however extends these approaches to be applicable to any two functionals without relying on pre-fitted energy correction parameters.

Using this framework, we propose two "mixing rules" that define our scheme for constructing mixed $\mathrm{r}^{2} \mathrm{SCAN} /$ GGA $(+U)$ phase diagrams. These mixing rules are summarized schematically in Figure 1 and briefly elaborated below. In sections that follow, we will illustrate each rule with an example.

1. Beginning with a $\mathrm{GGA}(+U)$ hull, replace $\mathrm{GGA}(+U)$ energies with $\mathrm{r}^{2} \mathrm{SCAN}$ energies by adding their $\Delta E_{\text {ref }}$ to the corresponding $\mathrm{GGA}(+U)$ reference energy.

2. Construct the convex energy hull using $\Delta H_{f}^{\mathrm{r}^{2} \mathrm{SCAN}}$ only when there are $\mathrm{r}^{2} \mathrm{SCAN}$ calculations corresponding to every reference structure (every $\mathrm{GGA}(+U)$ stable structure). In this case, add any missing GGA $(+U)$ materials by adding their $\Delta E_{\text {ref }}$ to the corresponding $\mathrm{r}^{2} \mathrm{SCAN}$ reference energy. 
Rule \#1 provides a means to introduce $\mathrm{r}^{2} \mathrm{SCAN}$ energies onto $\mathrm{GGA}(+U)$ phase diagrams when $\mathrm{r}^{2} \mathrm{SCAN}$ calculations are only available for one or a few compositions. In Figure 1, polymorph A represents the reference structure $(\mathrm{GGA}(+U)$ ground state). Since the reference structure is, by definition, on the $\mathrm{GGA}(+U)$ hull, the $\mathrm{r}^{2} \mathrm{SCAN}$ relaxed structure corresponding to this reference structure (as determined by the PYMATGEN [36] StructureMatcher algorithm) is assigned $\Delta H_{f}^{\mathrm{GGA}(+U)}$. Polymorphs B and C, calculated in $\mathrm{r}^{2} \mathrm{SCAN}$, are assigned energies that maintain their energy difference with respect to the reference structure, $\Delta E_{\text {ref }}$. For example, if polymorph $\mathrm{C}$ is $10 \mathrm{meV} /$ atom higher in energy than polymorph $\mathrm{A}$ in $\mathrm{r}^{2} \mathrm{SCAN}$, it would be assigned an energy $\Delta H_{f}^{\mathrm{GGA}(+U)}+10 \mathrm{meV} /$ atom. It is also possible for this method to place a polymorph below the $\mathrm{GGA}(+U)$ hull. Polymorph B is unstable with respect to the reference structure in $\mathrm{GGA}(+U)$ but is lower in energy than the reference structure in $\mathrm{r}^{2}$. If it were $10 \mathrm{meV} /$ atom lower than the reference structure in $\mathrm{r}^{2} \mathrm{SCAN}$, it would be assigned an energy $\Delta H_{f}^{\mathrm{GGA}(+U)}-10 \mathrm{meV} /$ atom, slightly changing the shape of the hull in the mixed phase diagram compared to GGA. Finally, polymorphs that do not have a $\mathrm{r}^{2} \mathrm{SCAN}$ energy (such as polymorph D) maintain their energy with respect to the $\mathrm{GGA}(+U)$ hull.

When $\mathrm{r}^{2} \mathrm{SCAN}$ calculations become available for every reference state, then, by Rule \#2, the convex hull is computed directly with $\mathrm{r}^{2} \mathrm{SCAN}$ formation energies $\left(\Delta H_{f}^{\mathrm{r}^{2} \mathrm{SCAN}}\right)$. Now, any unstable $\mathrm{GGA}(+U)$ phases that have not been calculated in $\mathrm{r}^{2} \mathrm{SCAN}$ can be added to the diagram by adding their $\Delta E_{\text {ref }}$ to the $H_{f}^{\mathrm{r}^{2} \mathrm{SCAN}}$ of the corresponding reference structure. In other words, we invert Rule \#1 so that $\mathrm{r}^{2} \mathrm{SCAN}$ structures become the reference structures.

Note that a central assumption of our mixing scheme is that $\mathrm{r}^{2} \mathrm{SCAN}$ energies are always preferable to GGA $(+U)$ energies. This assumption is well-justified by the generally superior accuracy of SCAN and $\mathrm{r}^{2} \mathrm{SCAN}$ formation energies reported in many studies [18, 22, 2731]. In general, application of our mixing scheme should be restricted to pairs of functionals where one has an $a$ priori reason to prefer one energy over another. In addition, we note that in principle it is possible to use our framework to mix energies from more than two functionals, provided that reference energies are available within each functional and that a clear hierarchy can be established among them.

\section{B. Mixed diagrams for relative polymorph stability (Rule \#1)}

We illustrate the motivation behind Rules \#1 and 2 using the $\mathrm{Sn}-\mathrm{Br}$ phase diagram, which is shown in Figure 2. In general, when constructing phase diagrams we seek to determine 1) the shape of the convex energy hull (i.e., stable compositions and their formation energies), and 2) the stable polymorph at each composition. Figure $2 \mathrm{a}$ and $2 \mathrm{~b}$ compare the $\mathrm{Sn}-\mathrm{Br}$ phase diagram with the formation energy of all phases calculated in $\mathrm{GGA}(+U)$ and $\mathrm{r}^{2} \mathrm{SCAN}$, respectively, and show that the accuracy of both aspects is improved by $\mathrm{r}^{2} \mathrm{SCAN}$. GGA $(+U)$ incorrectly predicts the ground-state polymorph of $\mathrm{SnBr}_{2}$ as rocksalt (spacegroup $P \overline{3} \mathrm{~m} 1$ ) and overpredicts the magnitude of $\Delta H_{f}$ as $-1.136 \mathrm{eV} /$ atom, whereas the experimental value is estimated at $-0.84-0.92 \mathrm{eV} /$ atom [37] (indicated by the shaded band in Figure 2). By contrast, $\mathrm{r}^{2} \mathrm{SCAN}$ correctly predicts the $\mathrm{SnBr}_{2}$ ground state as Pnma [28] and makes a substantially more accurate prediction of its formation energy $(-0.833 \mathrm{eV} /$ atom).

As we have discussed, it is not always feasible to recompute an entire chemical system using $\mathrm{r}^{2} \mathrm{SCAN}$ (as we have done to construct Figure 2b). When improving predictions of polymorph stability is a primary research objective, it makes sense to prioritize $\mathrm{r}^{2} \mathrm{SCAN}$ calculations for all known polymorphs at the composition of interest. However, if we were to apply this strategy to $\mathrm{SnBr}_{2}$ and replace all $\mathrm{GGA}(+U)$ formation energies of $\mathrm{SnBr}_{2}$ polymorphs with $\mathrm{r}^{2} \mathrm{SCAN}$ energies using naive mixing (Figure 2c), $\mathrm{SnBr}_{2}$ would no longer be predicted as stable. This occurs because the entire hull is shallower (smaller magnitude of $\Delta H_{f}$ ) in $\mathrm{r}^{2} \mathrm{SCAN}$ than in $\operatorname{GGA}(+U)$, and hence using an $\mathrm{r}^{2} \mathrm{SCAN}$ formation energy for $\mathrm{SnBr}_{2}$ causes it to move off the hull. Instead, we must apply Rule \#1 to make the $\mathrm{r}^{2} \mathrm{SCAN}$ energies compatible with the $\mathrm{GGA}(+U)$ hull. We do so by positioning $\mathrm{r}^{2} \mathrm{SCAN}$ formation energies relative to the $\mathrm{GGA}(+U)$ ground state polymorph $(P \overline{3} m 1)$, as shown in Figure $2 \mathrm{~d}$. Because we maintain the energy differences relative to this reference energy, the correct polymorph is now stabilized.

Compared to naive mixing of formation energies, applying Rule \#1 preserves the overall shape of the $\mathrm{GGA}(+U)$ convex hull while enabling improvement in phase stability predictions using as few as two $\mathrm{r}^{2} \mathrm{SCAN}$ calculations (one for the polymorph of interest and one for the reference structure). However, because $\mathrm{r}^{2} \mathrm{SCAN}$ stabilizes the Pnma polymorph instead of the $P \overline{3} m 1$ polymorph stabilized by $\mathrm{GGA}(+U), \Delta H_{f}$ is lowered (and made less accurate) by $37 \mathrm{meV} /$ atom, which is the difference in energy between the $P \overline{3} m 1$ and $P n m a$ polymorphs in $\mathrm{r}^{2} \mathrm{SCAN}$. Hence, although use of Rule \#1 for study of a single composition may yield more accurate relative polymorph energies, it carries the risk of making the magnitude of the formation energy slightly less accurate compared to a full $\mathrm{r}^{2} \mathrm{SCAN}$ phase diagram.

\section{Mixed diagrams for formation energy (Rule \#2)}

When identifying stable compositions or predicting accurate formation energies is the primary research objective, it makes sense to prioritize recomputing all $\mathrm{GGA}(+U)$ ground states in $\mathrm{r}^{2} \mathrm{SCAN}$, as shown in Figure 2e. Applying Rule \#2, this strategy allows the entire 

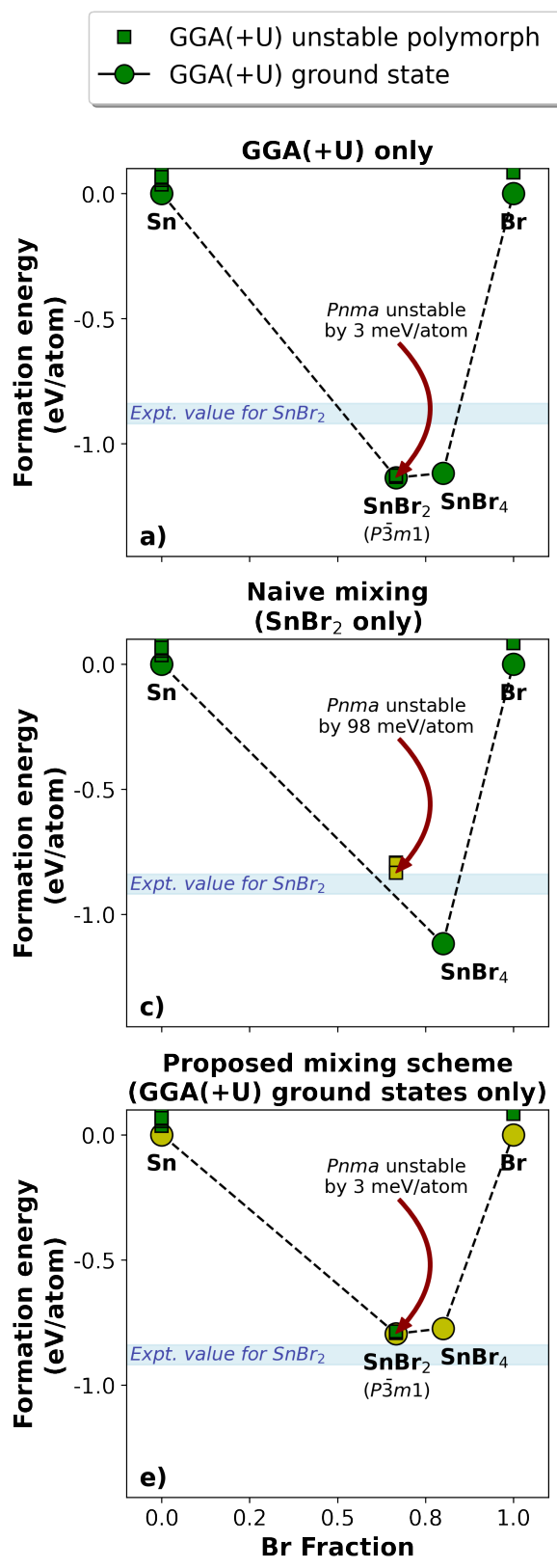

$\begin{array}{ll}\square & r^{2} \text { SCAN unstable polymorph } \\ -0 & r^{2} \text { SCAN ground state }\end{array}$

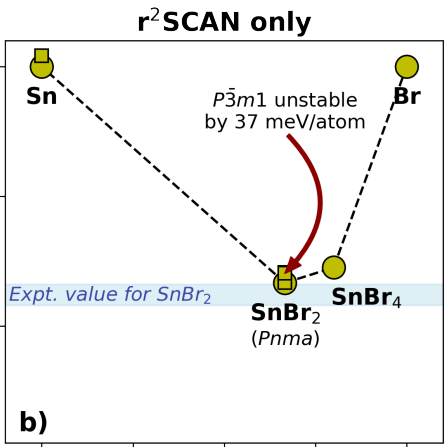

Proposed mixing scheme $\left(\mathrm{SnBr}_{2}\right.$ only)

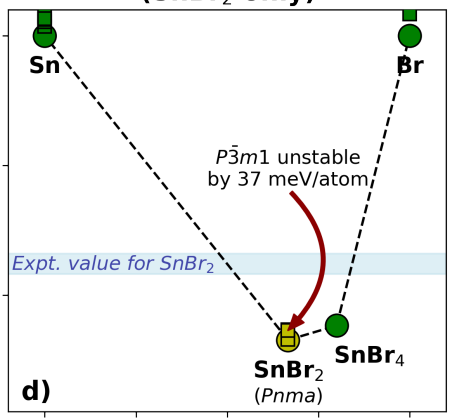

Proposed mixing scheme (all $<20 \mathrm{meV} /$ atom)

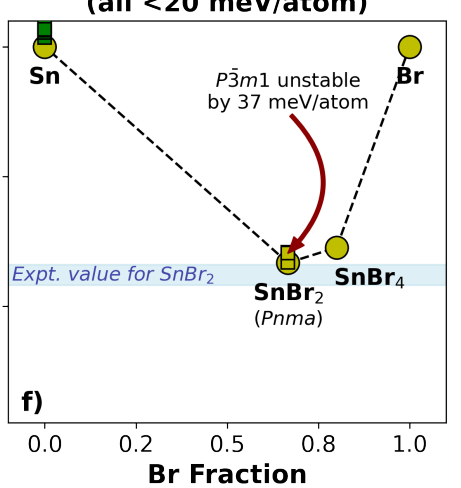

FIG. 2. Sn-Br phase diagram constructed using different mixing strategies for $\mathrm{GGA}(+U)$ and $\mathrm{r}^{2} \mathrm{SCAN}$ calculations. a) $\mathrm{GGA}(+U)$ only; b) $\mathrm{r}^{2} \mathrm{SCAN}$ only; c) naive mixing of formation energies, where all $\mathrm{SnBr}_{2}$ polymorphs are computed in $\mathrm{r}^{2} \mathrm{SCAN}$ and all other materials are in GGA; d) the same set of energies as c), but employing our mixing scheme (Rule \#2); e) $\mathrm{r}^{2} \mathrm{SCAN}$ for all reference states (i.e., $\mathrm{GGA}(+U)$ ground states) and $\mathrm{GGA}(+U)$ for all other materials; f) $\mathrm{r}^{2} \mathrm{SCAN}$ for all materials within $20 \mathrm{meV} /$ atom of the GGA $(+U)$ convex hull. The numerical value in parentheses indicates the energy above hull of the experimental ground state Pnma polymorph of $\mathrm{SnBr}_{2}$. The shaded blue regions represent the estimated range of experimental formation energies for $\mathrm{SnBr}_{2}$ [37]. Tabulated $\mathrm{r}^{2} \mathrm{SCAN}$ and $\mathrm{GGA}(+U)$ energies for all materials are provided in Appendix E.

convex hull to be constructed using $\Delta H_{f}^{\mathrm{r}^{2} \mathrm{SCAN}}$. Unstable $\mathrm{GGA}(+U)$ polymorphs are then positioned relative to the corresponding reference structures. Several unstable polymorphs of Sn that were mixed in this manner are visible in Figure 2e.

In this chemical system, recomputing the hull when only the $\mathrm{GGA}(+U)$ ground states have been calculated in $\mathrm{r}^{2} \mathrm{SCAN}$ will still not recover the exact $\mathrm{r}^{2} \mathrm{SCAN}$ for- mation energy for $\mathrm{SnBr}_{2}$, because $\mathrm{GGA}(+U)$ stabilizes the incorrect ground state, and Rule \#2 treats this incorrect ground state as the reference energy. Hence, the formation energy of $\mathrm{SnBr}_{2}$ predicted by the mixed phase diagram in Figure 2e is too high by $37 \mathrm{meV} /$ atom (the difference in energy between the $P \overline{3} m 1$ and Pnma polymorphs in $\left.\mathrm{r}^{2} \mathrm{SCAN}\right)$.

Because there is no way to know a priori whether 
$\mathrm{r}^{2} \mathrm{SCAN}$ will stabilize a different ground state than $\mathrm{GGA}(+U)$, a more robust strategy is to compute all polymorphs within some tolerance of the GGA $(+U)$ hull. Computing both ground states and slightly metastable polymorphs with $\mathrm{r}^{2} \mathrm{SCAN}$ makes it more likely that the shape of the convex energy hull in the mixed phase diagram will be identical to that in a full $\mathrm{r}^{2}$ SCAN diagram. We apply this strategy in Figure 2f, in which we mix $\mathrm{r}^{2} \mathrm{SCAN}$ energies for all materials within $20 \mathrm{meV} /$ atom of the GGA $(+U)$ hull, and use $\mathrm{GGA}(+U)$ energies for all other materials. The value of $20 \mathrm{meV} /$ atom is motivated by analysis presented later (see Section III B) indicating that materials with higher $\Delta E_{\mathrm{hull}}^{\mathrm{GGA}(+U)}$ are rarely stabilized by $\mathrm{r}^{2} \mathrm{SCAN}$. With this strategy, the shape of the resulting energy hull (Figure 2f) exactly matches that of the pure $\mathrm{r}^{2} \mathrm{SCAN}$ hull (Figure $2 \mathrm{~b}$ ).

Comparing Figures 2a, 2c, and 2e illustrates the importance of Rule \#2 when mixing $\mathrm{r}^{2} \mathrm{SCAN}$ and GGA $(+U)$ calculations. Formation energies cannot be naively mixed without carrying a substantial risk of over- or understabilizing certain compositions. The hull must remain in $\operatorname{GGA}(+U)$ until there are $\mathrm{r}^{2}$ SCAN calculation corresponding to every reference state. Even in that case, it is preferable to include slightly unstable polymorphs in order to achieve better accuracy in cases where $\mathrm{r}^{2} \mathrm{SCAN}$ stabilizes different polymorphs.

\section{PRACTICAL CONSIDERATIONS}

\section{A. Mixed diagrams for ternary and higher systems}

Ternary or higher-dimensional chemical spaces present special challenges for mixing energies between functionals, because strict application of mixing rules \#1 and \#2 can introduce inconsistencies between the full phase diagrams and those of constituent subsystems. For example, consider a case in which all of the GGA $(+U)$ ground states in chemical system A-B are computed in $\mathrm{r}^{2} \mathrm{SCAN}$. According to Rule \#2, the binary A-B phase diagram would be constructed using $\Delta H_{f}^{\mathrm{r}^{2} \mathrm{SCAN}}$. This may result in different formation energies and/or predicted stable phases than the GGA $(+U)$ phase diagram, as illustrated previously for the $\mathrm{Sn}-\mathrm{Br}$ system. Now suppose that we wish to construct a ternary phase diagram for the A-B-C system, in which there are multiple ternary ground states that have not been computed in $\mathrm{r}^{2} \mathrm{SCAN}$. Since the AB-C system does not satisfy the requirements for Rule \#2, we would construct this ternary phase diagram using $\Delta H_{f}^{\mathrm{GGA}(+U)}$. This could result in the ternary A-B$\mathrm{C}$ diagram predicting different formation energies and/or stable phases in the A-B subsystem than the binary AB diagram. Such an inconsistency may be problematic depending on the use case. Note that if all reference energies in the full $\mathrm{A}-\mathrm{B}-\mathrm{C}$ system have been recomputed in $\mathrm{r}^{2} \mathrm{SCAN}$, then strict application of the mixing rules will not result in any inconsistencies. However, due to the much larger number of ternary and higher materials (compared to binaries), it becomes progressively more difficult to recompute all the reference energies needed to apply Rule \#2 as the size of the chemical system increases.

In cases where consistency between lower- and higherdimension phase diagrams is essential, one may apply the mixing rules individually to each chemical subsystem, in order of increasing dimensionality. To continue the example above, Rule \#1 and Rule \#2 would be applied individually to each of the $\mathrm{A}-\mathrm{B}, \mathrm{B}-\mathrm{C}$, and $\mathrm{A}-\mathrm{C}$ chemical systems. The ternary phase diagram would then be constructed by combining these pre-adjusted binary formation energies with $\mathrm{GGA}(+U)$ formation energies. An example of such a diagram is presented in Figure C.2.

Applying the mixing scheme to binary subsystems before treating the ternary system amounts to a modified form of naive mixing because it involves directly combining formation energies obtained from $\mathrm{GGA}(+U)$ (for ternaries) with those calculated with $\mathrm{r}^{2} \mathrm{SCAN}$ for binaries, without considering whether $\mathrm{r}^{2} \mathrm{SCAN}$ energies are available for all ternary ground states. As such, mixed phase diagrams for high-dimensional chemical systems that are constructed in this manner should be used sparingly and interpreted with care. However, due to the inherently larger number of phases involved in higher dimensional systems, we expect this modified form of naive mixing to be less likely to cause severe distortions of the hull compared to binary systems.

To test this hypothesis, we compared ternary phase stability predictions from approximately 6,000 ternary phase diagrams computed in $\mathrm{GGA}(+U)$ to mixed versions constructed using $\Delta H_{f}^{\mathrm{r}^{2} \mathrm{SCAN}}$ values for all binary subsystems and $\Delta H_{f}^{\mathrm{GGA}(+U)}$ for all ternary materials (see Appendix C). We evaluated how frequently these "edged" diagrams either 1) destabilized a known experimental ternary phase (i.e., a phase reported in the Inorganic Crystal Structure Database [38]) that was stable in pure $\mathrm{GGA}(+U)$ or 2$)$ stabilized a known experimental ternary phase that was unstable in pure GGA $(+U)$. For the majority of chemical systems (83\%), experimental ternary materials predicted stable by the pure $\mathrm{GGA}(+U)$ diagram remained so in the mixed phase diagram, while in another $14 \%$ of cases exactly one material was destabilized. Similarly, for $94 \%$ of chemical systems, experimental materials predicted unstable by the pure $\mathrm{GGA}(+U)$ diagram remain so in the mixed diagram, while for $6 \%$ of chemical systems exactly one unstable experimental material was stabilized (see Figure C.1). Thus, although employing modified naive mixing (i.e., "edged" phase diagrams) to achieve consistency between lower- and higher-dimensional phase diagrams carries a modest risk of destabilizing known experimental phases for some chemical systems, there are many other cases in which the mixed diagrams stabilize experimental phases that pure $\mathrm{GGA}(+U)$ does not. Altogether, these results suggest that modified naive mixing is unlikely to severely 
distort phase stability predictions.

\section{B. Definition of materials "close to the hull"}

In Section II we observed that it is preferable to recompute not just GGA $(+U)$ ground states, but also materials close to the convex energy hull in order to ensure that the mixed energy hull has the correct shape (compare Figures 2e and $\mathrm{f}$ ). This begs the question of how to define "close to the hull". More specifically, we can rephrase the question as "how likely is $\mathrm{r}^{2} \mathrm{SCAN}$ to stabilize a material that is $\mathrm{X} \mathrm{meV} /$ atom above the hull in GGA $(+U)$ ?" For example, a material that is 500 $\mathrm{meV}$ /atom above hull in GGA $(+U)$ will almost certainly not become stable in $\mathrm{r}^{2} \mathrm{SCAN}$, but a material that is unstable by $3 \mathrm{meV} /$ atom could (as was the case with Pnma $\mathrm{SnBr}_{2}$ in Section II). Determining an appropriate threshold is necessary to properly target $\mathrm{r}^{2} \mathrm{SCAN}$ calculations.

To inform this question, in Appendix A we evaluate the extent to which $\mathrm{r}^{2} \mathrm{SCAN}$ changes the energy above hull of unstable polymorphs. Examining approximately 7300 unstable materials with a GGA $+(U)$ energy above hull of $50 \mathrm{meV} /$ atom or less, we find that in $95 \%$ of cases, the energy above hull either increases or decreases by no more than $19 \mathrm{meV} /$ atom (see Figure A.1). This means that materials more than $19 \mathrm{meV} /$ atom above the $\mathrm{GGA}(+U)$ hull would only be stabilized by $\mathrm{r}^{2} \mathrm{SCAN}$ in rare cases. Hence, we adopt a threshold of $20 \mathrm{meV} /$ atom as our definition of "close to the hull" for purposes of prioritizing calculations.

By way of comparison, we note that among 16 systems identified by Yang et al. [28] in which SCAN stabilized the correct ground state and GGA $(+U)$ did not, the energies above hull of the experimental ground states in $\mathrm{GGA}(+U)$ ranged from 2 to $50 \mathrm{meV} /$ atom. Another study showed that SCAN mispredicted the ground states of $\mathrm{TiO}_{2}$ and $\mathrm{FeS}_{2}$, with misprediction on the order of 50 $\mathrm{meV} /$ atom as well [39]. Thus, although based on analysis of a large set of materials, our selection of $20 \mathrm{meV} /$ atom as a "safe" threshold is not guaranteed to capture the $\mathrm{r}^{2} \mathrm{SCAN}$ ground state polymorph in every case. A higher threshold could certainly be chosen if greater confidence in capturing the correct ground states is required.

\section{Failures of structure matching}

In Section IIA we established the need to obtain $\mathrm{r}^{2} \mathrm{SCAN}$ energies of $\mathrm{GGA}(+U)$ ground states, which serve as reference energies for constructing mixed phase diagrams. To obtain the most accurate $\mathrm{r}^{2} \mathrm{SCAN}$ energies, we generally perform $\mathrm{r}^{2} \mathrm{SCAN}$ structure optimizations rather than single-point calculations and then use the PYMATGEN [36] StructureMatcher algorithm to determine whether the $\mathrm{r}^{2} \mathrm{SCAN}$-relaxed structure is the same (within tolerances) as the $\mathrm{GGA}(+U)$ starting structure.
In the vast majority of cases, the $\mathrm{r}^{2} \mathrm{SCAN}$-relaxed structure and the GGA $(+U)$ starting structure match, allowing us to use the $\mathrm{r}^{2} \mathrm{SCAN}$ energy as a reference energy. However in selected cases (some $1 \%$ of all materials we have computed thus far), $\mathrm{r}^{2} \mathrm{SCAN}$ will optimize to a structure that is no longer considered equivalent to the starting structure. This is especially common for the crystal structures of diatomic molecules (e.g., $\mathrm{H}_{2}$, $\mathrm{Cl}_{2}, \mathrm{O}_{2}$ ) in which the different treatment of short- and medium-range interactions by ${ }^{2} \mathrm{SCAN}$ compared to $\mathrm{PBE}$ is particularly significant.

We address this issue in two ways. In some cases, manual inspection of the structures allows us to establish that they represent the same material, and hence that the $\mathrm{r}^{2} \mathrm{SCAN}$ energy can be used as a reference energy. However, manual inspection is not feasible for highthroughput work. Instead, we perform single-point calculations for any materials in which the $\mathrm{r}^{2} \mathrm{SCAN}$-relaxed structure no longer matches the input structure. The $\mathrm{r}^{2} \mathrm{SCAN}$ single-point calculation is guaranteed to match the corresponding $\mathrm{GGA}(+U)$-optimized structure and provides an $\mathrm{r}^{2} \mathrm{SCAN}$ energy that can serve as a reference energy. Meanwhile, an $\mathrm{r}^{2} \mathrm{SCAN}$ optimization of the same structure (which may no longer be the same according to the StructureMatcher) is guaranteed to have a similar or lower energy than the single point and will be added to the hull at the correct position by application of Rule \#1. Performing $\mathrm{r}^{2} \mathrm{SCAN}$ single points also provides a means of obtaining reference energies for large structures that would be impractical to optimize in $\mathrm{r}^{2} \mathrm{SCAN}$ within reasonable computational limits (see Section IIID).

\section{Prioritizing $\mathbf{r}^{2} \mathbf{S C A N}$ calculations for maximum benefit}

We conclude our discussion of practical considerations by considering the best strategy for prioritizing $\mathrm{r}^{2} \mathrm{SCAN}$ calculations, given that computational resources are limited and that its cost is still approximately $5 \times$ that of PBE [18].

We can define several levels of "calculation coverage" (meaning, subsets of materials that have all been recomputed with $\mathrm{r}^{2} \mathrm{SCAN}$, Figure 3 ) based on the mixing rules we have established. In order to apply Mixing Rule \#1, at least two $\mathrm{r}^{2} \mathrm{SCAN}$ optimizations at a single composition are needed: one for the $\mathrm{GGA}(+U)$ ground state and one for another polymorph. To apply Rule \#2, we require $\mathrm{r}^{2} \mathrm{SCAN}$ energies for every $\mathrm{GGA}(+U)$ ground state or (ideally) every $\mathrm{GGA}(+U)$ material within 20 $\mathrm{meV} /$ atom of the hull. These energies are preferably obtained from structure optimizations, although as discussed above, single-point calculations can be used, with the risk of a slightly less accurate hull shape. The pinnacle of calculation coverage (which may have less value that its computational cost, as noted in the Introduction) is full recomputation of all materials using $\mathrm{r}^{2} \mathrm{SCAN}$.

With a goal of achieving second- or third-level cov- 


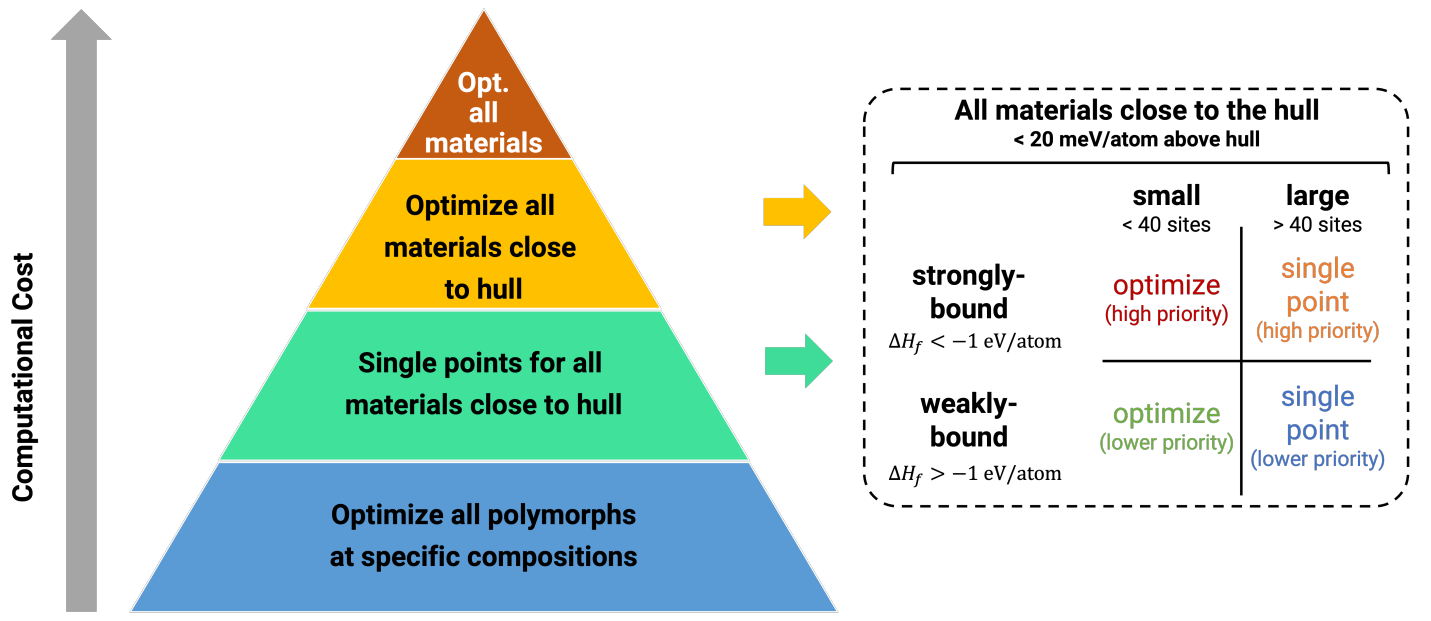

FIG. 3. Strategies for prioritizing higher level calculations in large materials databases. Left: different stages of calculation coverage. The first level enables application of Mixing Rule \#1, while the second and third levels facilitate application of Rule \#2. The fourth level (re-optimizing all materials with higher level calculations) is not necessary. Right: Methods used by the Materials Project to pursue second- and third-level coverage of $\mathrm{r}^{2} \mathrm{SCAN}$ calculations. Optimizations of small, strongly-bound materials have the highest priority. Single-point calculations are used for large structures. Weakly-bound materials have lower priority. See Section IIID for further details.

erage, we can identify several strategies for prioritizing which materials to calculate in order to maximize the benefits of the mixing scheme for formation energy prediction. To do so, we classify materials close to the hull as 1) strongly- or weakly-bound and 2) small or large.

Previous studies [18, 22] established that SCAN and $\mathrm{r}^{2} \mathrm{SCAN}$ predict substantially more accurate formation energies than PBE or PBEsol for "strongly-bound" materials, i.e., materials whose $\mathrm{GGA}(+U)$-predicted formation energy is lower than $-1 \mathrm{eV} /$ atom. The improvement in accuracy for "weakly-bound" materials is more modest. As such, creating mixed phase diagrams for stronglybound systems is likely to improve overall accuracy the most, and hence we assign higher priority to stronglybound materials.

With respect to size, experience indicates that optimizations of large structures (e.g., larger than approximately 40 sites) with $\mathrm{r}^{2}$ SCAN will often exceed typical maximum wall time limits at supercomputing centers (e.g. $48 \mathrm{hr}$ ). This is not to say optimization is impossible; rather, in a high-throughput computing context it does not usually make sense to invest an excessive amount of computing nodes or wall time into a single material. As such, we choose to perform single-point calculations for large materials in order to obtain a reference energy (albeit a less accurate one) so that Rule \#2 can be applied. Fully-optimized structures can be obtained as computational resources allow, and added into the mixed phase diagrams according to Rule \#1.

\section{EXAMPLES}

\section{A. Application to a metastable ternary nitride system}

As a practical example of our complete mixing scheme, we use it to investigate compound metastability in the ternary Zn-Sb-N system. Nitrides remain relatively unexplored compared to other chemical spaces, even though they exhibit the largest range of thermodynamicallyaccessible metastable states among inorganic materials $[42,43]$, which is thought to be a consequence of the large cohesive energy of metal-nitrogen bonds that kinetically traps metastable structures [40]. Compared to stable nitrides, metastable nitrides are more likely to contain metal cations in high oxidation states, which imparts unique semiconducting properties that make these materials interesting for electronic and photovoltaic applications, among others [29, 40, 43].

Metastable nitrides are relatively rare in nature and difficult to synthesize experimentally due to the high stability of molecular $\mathrm{N}_{2}$. However, the use of reactive nitrogen precursors such as ammonia, azide compounds, or plasma-cracked atomic $\mathrm{N}$ allow nitrogen chemical potentials of up to $+1 \mathrm{eV} / \mathrm{N}$ above the hull to be reached in laboratory synthesis $[29,40,41]$. Recent experimental studies [44-46] have reported synthesis of several metastable nitrides $\left(\mathrm{Cu}_{3} \mathrm{~N}, \mathrm{Sn}_{3} \mathrm{~N}_{4}\right.$, and Tialloyed $\mathrm{Sn}_{3} \mathrm{~N}_{4}$ ). Ternary Wurtzite-based nitrides, such as $\mathrm{MgSnN}_{2}, \mathrm{ZnSnN}_{2}, \mathrm{ZnGeN}_{2}$, and $\mathrm{ZnSiN}_{2}$, have received specific attention recently as potential alternatives to III$\mathrm{V}$ semiconductors. [41, 44]. Computational screening studies $[29,40]$ recently predicted three new metastable ternary phases $\left(\mathrm{ZnSb}_{2} \mathrm{~N}_{4}, \mathrm{Zn}_{2} \mathrm{SbN}_{3}\right.$, and $\left.\mathrm{Zn}_{3} \mathrm{SbN}_{3}\right)$ and 


\begin{tabular}{rlrl}
$\square$ & GGA $(+\mathrm{U})$ unstable & & $\mathrm{r}^{2}$ SCAN unstable \\
$-\mathrm{GGA}(+\mathrm{U})$ metastable $(\Delta \mu \leq 1 \mathrm{eV} / \mathrm{N})$ & & $\mathrm{r}^{2}$ SCAN metastable $(\Delta \mu \leq 1 \mathrm{eV} / \mathrm{N})$ \\
$-\mathrm{GGA}(+\mathrm{U})$ ground state & - & - & $\mathrm{r}^{2}$ SCAN ground state \\
\hline
\end{tabular}

\section{GGA(+U) only}

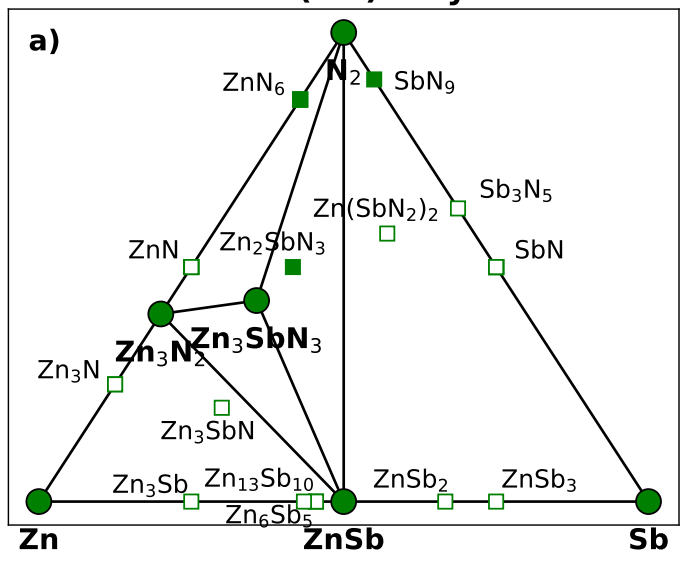

$r^{2}$ SCAN edges (strict mixing rules)

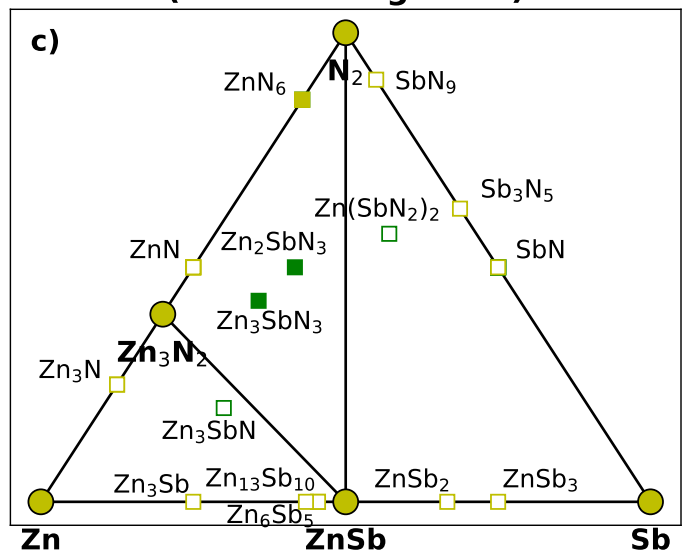

$\mathbf{r}^{2}$ SCAN only
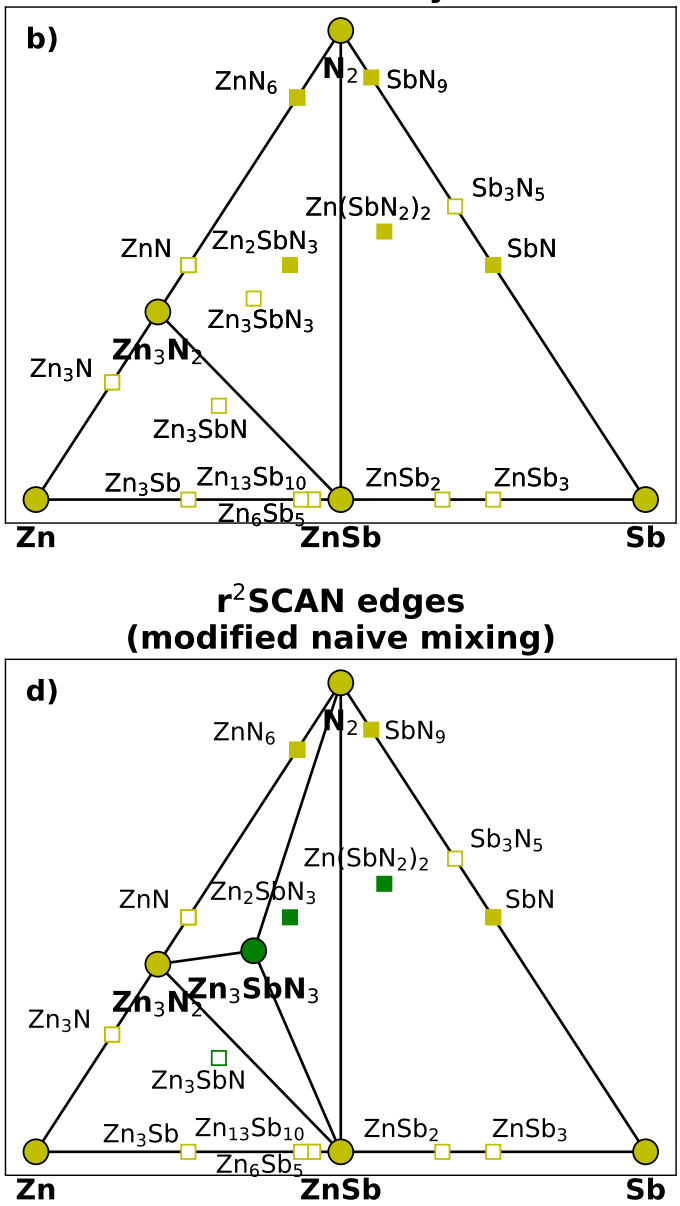

FIG. 4. Zn-Sb-N phase diagrams illustrating different mixing strategies for $\mathrm{GGA}(+U)$ and $\mathrm{r}^{2} \mathrm{SCAN}$ calculations. a) GGA(+ $\left.U\right)$ only; b) $\mathrm{r}^{2}$ SCAN only; c) strict application of Rule \#1 and \#2 to calculations comprising $\mathrm{r}^{2}$ SCAN energies for all elements and binary phases with $\mathrm{GGA}(+U)$ energies for all ternary phases d) same set of calculations as c), but using modified naive mixing in which binary hulls are constructed from $\mathrm{r}^{2} \mathrm{SCAN}$ formation energies. Phases labeled "metastable" are phases that can be stabilized by a $+1 \mathrm{eV} / \mathrm{N}$ increase in the nitrogen chemical potential, which is achievable in laboratory synthesis [29, 40, 41]. Tabulated $\mathrm{r}^{2} \mathrm{SCAN}$ and $\mathrm{GGA}(+U)$ energies for all materials are provided in Appendix E.

a new metastable binary phase $(\mathrm{SbN})$ in the $\mathrm{Zn}-\mathrm{Sb}-$ $\mathrm{N}$ chemical space. $\mathrm{Zn}_{2} \mathrm{SbN}_{3}$, the first Sb-based nitride semiconductor ever reported, was experimentally realized [40, 41, 47] and exhibited promising electronic properties for photovoltaic and water splitting applications. $\mathrm{SbN}$ was predicted to be relatively close to the metastability limit (requiring $+0.8 \mathrm{eV} / \mathrm{N}$ to stabilize) [29] and is the subject of ongoing investigations as another potential Sb-based nitride semiconductor.

Given the diverse bonding characteristics of nitrogen compounds, computational predictions of metastability can be particularly sensitive to the choice of functional and energy correction scheme (e.g., GGA vs. GGA+U vs. $\left.\mathrm{r}^{2} \mathrm{SCAN}\right)$. For example, Sun et al. [29] showed that for many binary nitride systems, PBE overstabilizes the nitrogen-rich region of the convex energy hull, while GGA + U overstabilized the nitrogen-poor region. SCAN was found to predict formation enthlapies with good accuracy across both portions of the hull [29]. To expand on this previous work and inform future high-throughput screening studies, we evaluate how the use of a mixed $\mathrm{r}^{2} \mathrm{SCAN} / \mathrm{GGA}(+U)$ ternary phase diagram would affect these predictions.

Figures $4 \mathrm{a}$ and $4 \mathrm{~b}$ show the phase diagrams computed entirely using $\mathrm{GGA}(+U)$ and $\mathrm{r}^{2} \mathrm{SCAN}$ calculations, respectively. Both the pure $\mathrm{GGA}(+U)$ and pure $\mathrm{r}^{2} \mathrm{SCAN}$ 
phase diagrams predict that $\mathrm{Zn}_{2} \mathrm{SbN}_{3}$ and $\mathrm{SbN}$ are metastable, consistent with the previous studies. However, the energy above hull of both metastable compositions of interest is higher in $\mathrm{r}^{2} \mathrm{SCAN}$. $\mathrm{r}^{2} \mathrm{SCAN}$ predicts $30 \mathrm{meV}$ /atom above hull for $\mathrm{Zn}_{2} \mathrm{SbN}_{3}$ (vs. $20 \mathrm{meV}$ /atom in GGA), reflecting the overstabilization of the N-rich region of the phase diagram in $\mathrm{GGA}(+U)$ noted by Sun et al. [29]. $\mathrm{r}^{2} \mathrm{SCAN}$ predicts $260 \mathrm{meV} /$ atom above hull for $\mathrm{SbN}$ (vs. $172 \mathrm{meV} /$ atom in GGA), but in the $\mathrm{r}^{2} \mathrm{SCAN}$ diagram this material falls within the metastable synthesizability limit, consistent with experimental reports [29]. The other notable differences between the $\mathrm{GGA}(+U)$ and $\mathrm{r}^{2} \mathrm{SCAN}$ phase diagrams are that $\mathrm{Zn}_{3} \mathrm{SbN}_{3}$, which has not been synthesized to the best of our knowledge and is not in the ICSD, is predicted stable by GGA $(+U)$ but unstable by $\mathrm{r}^{2} \mathrm{SCAN}$, while $\mathrm{ZnSb}_{2} \mathrm{~N}_{4}$ is unstable in $\operatorname{GGA}(+U)$ but metastable in $\mathrm{r}^{2} \mathrm{SCAN}$. The stable / metastable / unstable classification for all other compositions is the same in both diagrams.

Moving to mixed phase diagrams, we now consider a situation in which only the elements and binary compositions have been computed with $\mathrm{r}^{2} \mathrm{SCAN}$, while all ternary phases remain in $\mathrm{GGA}(+U)$. We compare two methods of constructing this mixed ternary phase diagram in Figures $4 \mathrm{c}$ and $4 \mathrm{~d}$. In Figure 4c, we apply Mixing Rule \#1 and \#2 strictly (i.e., considering the entire phase diagram at once). In this scenario, because we do not have a $\mathrm{r}^{2} \mathrm{SCAN}$ calculation for the $\mathrm{GGA}(+U)$ reference structure $\mathrm{Zn}_{3} \mathrm{SbN}_{3}$, the hull is still calculated using $G G A(+U)$ energies (with the exception of polymorphs stablized by $\mathrm{r}^{2} \mathrm{SCAN}$, as discussed later). $\mathrm{r}^{2} \mathrm{SCAN}$ polymorphs for each element or binary composition are placed on this $\mathrm{GGA}(+U)$ hull by anchoring to the respective reference states according to Rule \#1.

Inspection of Figure 4c shows broad similarity to the pure $\mathrm{r}^{2} \mathrm{SCAN}$ diagram (Figure $4 \mathrm{~b}$ ), with a few notable differences. $\mathrm{Zn}_{3} \mathrm{SbN}_{3}$ is predicated unstable in the pure $\mathrm{r}^{2} \mathrm{SCAN}$ diagram yet metastable in the mixed diagram. For $\mathrm{SbN}$ the reverse is true: this material is predicted metastable in the pure $r^{2} \mathrm{SCAN}$ diagram but unstable in this mixed diagram. The convex energy hull in Figure 4c is constructed with $\mathrm{GGA}(+U)$ energies, so it is identical to that of Figure $4 \mathrm{a}$ with one significant exception. As noted in Section IIB, Rule \#1 can cause the energy of the convex hull to decrease in cases where $\mathrm{r}^{2} \mathrm{SCAN}$ stabilizes a different polymorph than $\mathrm{GGA}(+U)$. In this case, $\mathrm{r}^{2} \mathrm{SCAN}$ stabilizes a different structure for $\mathrm{N}_{2}$ (which is a crystalline solid at $0 \mathrm{~K}$ ) that is $1.8 \mathrm{meV} /$ atom lower in energy than the reference energy (see Figure B.1), causing it to be placed below the $\operatorname{GGA}(+U)$ hull and thereby "lowering" the hull energy of the N-rich region in the mixed phase diagram. This causes $\mathrm{Zn}_{3} \mathrm{SbN}_{3}$, which is predicted stable in the pure $\mathrm{GGA}(+U)$ phase diagram, to move off the hull by $0.4 \mathrm{meV} /$ atom and become metastable. The energy above hull for SbN increases by $1 \mathrm{meV} /$ atom, and hence it retains its classification as unstable consistent with the $\mathrm{GGA}(+U)$ phase diagram.

(Figure 4d) presents an alternative phase diagram con- structed by fully applying Rules \#1 and \#2 to the binary edges and then adding $\mathrm{GGA}(+U)$ energies for ternary phases by modified naive mixing, as discussed in Section III A. Here, the edges of the diagram are identical to those predicted by a pure $\mathrm{r}^{2} \mathrm{SCAN}$ diagram because we have full coverage of all $\mathrm{GGA}(+U)$ ground states and hence Rule \#2 applies. In the interior of the diagram, three of the four ternary compositions retain the same stable/unstable/metastable classification they have in the pure $\mathrm{r}^{2} \mathrm{SCAN}$ diagram, while $\mathrm{Zn}_{3} \mathrm{SbN}_{3}$ is predicted to be stable in the $\mathrm{GGA}(+U)$ diagram (whereas it is predicted unstable in the pure $\mathrm{r}^{2} \mathrm{SCAN}$ diagram).

As noted in Section III A, the modified form of naive mixing employed to construct Figure $4 \mathrm{~d}$ is thermodynamically less consistent than strict application of Rule \#1 and \#2 (Figure 4c), and should only be invoked when consistency between binary and higher dimension phase diagrams is essential. In this example, where the metastability of phases is of primary interest, it would be advisable to apply the mixing rules strictly to ensure that the entire convex hull is constructed in a consistent manner. Indeed, among the two mixed diagrams, Figure 4c shows the most consistency with the pure $\mathrm{r}^{2} \mathrm{SCAN}$ diagram.

\section{B. Application to aqueous phase stability}

Finally, we demonstrate how the mixing scheme presented here can be used to inform aqueous phase stability predictions. The computational Pourbaix diagram formalism of Persson et al. [35, 48] generates aqueous stability ( $\mathrm{pH}-\mathrm{pE}$ ) diagrams by referencing experimental free energies of dissolved ions to DFT-predicted formation energies derived from solid phase diagrams. As such, the mixing scheme presented here can be applied to the creation of Pourbaix diagrams in addition to solid phase diagrams. SCAN-derived Pourbaix diagrams, for example, were shown to be systematically more accurate for transition metal oxides [49]. However, the large number of stable phases needed to build computational Pourbaix diagrams may preclude calculating entire chemical spaces in SCAN or $\mathrm{r}^{2} \mathrm{SCAN}$, motivating the usefulness of our mixing scheme in this context.

We illustrate the mixing scheme on the Se-O system, for which the PBE-derived Pourbaix diagram is known to be inaccurate with respect to experiment [49]. Specifically, it predicts a stable $\mathrm{SeO}_{2}$ phase that is not observed experimentally (Figure 5a). Creating a computational Pourbaix diagram of this system requires a solid phase diagram of the $\mathrm{Se}-\mathrm{O}-\mathrm{H}$ chemical system, which contains 85 individual materials, according to the Materials Project database. Five of these materials contain more than 40 sites, and hence could be particularly challenging to recompute in $\mathrm{r}^{2} \mathrm{SCAN}$ (see Section IIID). Use of the mixing scheme allows us to construct the hull in $\mathrm{r}^{2} \mathrm{SCAN}$ by performing calculations only for the ground states $(9$ materials) while retaining information from $\mathrm{GGA}(+U)$ 

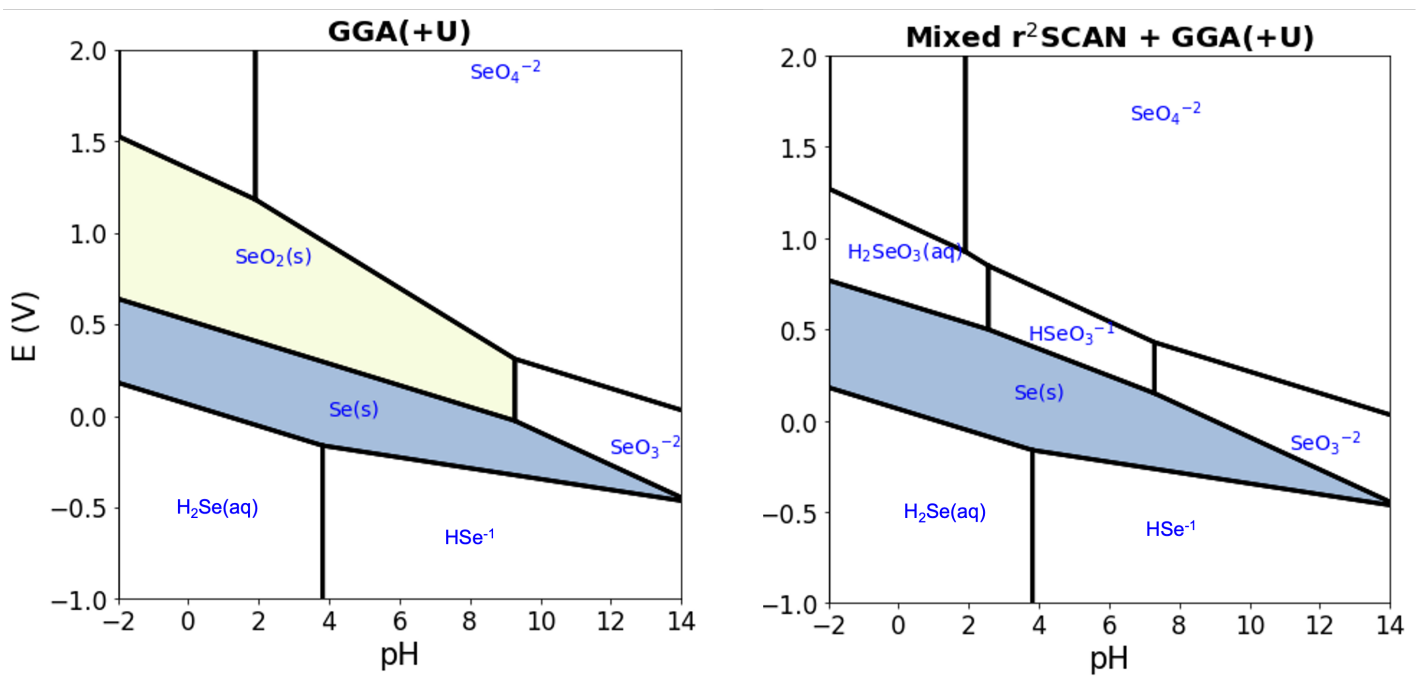

FIG. 5. Pourbaix diagram of Se-O constructed using GGA $(+U)$ calculations (left) and the mixing scheme presented in this work (right) at a total Se concentration of $10^{-6} \mathrm{~mol} / \mathrm{L}$. Shaded regions predict stable solid phases. Tabulated $\mathrm{r}^{2} \mathrm{SCAN}$ and $\mathrm{GGA}(+U)$ energies for all materials are provided in Appendix E.

about the metastability of other phases.

The resulting Pourbaix diagram built from mixed $\mathrm{r}^{2} \mathrm{SCAN}$ and $\mathrm{GGA}(+U)$ energies (Figure $\left.5 \mathrm{~b}\right)$ correctly predicts that the oxide phase $\mathrm{SeO}_{2}$ is unstable (unlike the pure $\mathrm{GGA}(+U)$ diagram), in agreement with Pourbaix diagrams presented by Wang et al. [49] that were prepared from both experimental data and pure SCAN calculations. Hence for this system, our mixing scheme has made it possible to leverage a relatively small number of calculations and achieve similar predictive accuracy as full recalculation of all materials with SCAN.

\section{Summary}

In summary, we have developed a mixing scheme to enable construction of phase diagrams that combine formation energies from any two DFT functionals. Such a capability is important to high-throughput materials screening efforts because it allows a relatively low-coverage, high-fidelity set of calculations (here, $\mathrm{r}^{2} \mathrm{SCAN}$ ) to be used in concert with existing high-coverage, lower-fidelity calculations (here, $\mathrm{GGA}(+U)$ ) to improve the accuracy of phase stability predictions. Our scheme allows mixing $\mathrm{r}^{2} \mathrm{SCAN}$ and $\mathrm{GGA}(+U)$ calculations when as few as two $\mathrm{r}^{2} \mathrm{SCAN}$ calculations (one corresponding to a $\mathrm{GGA}(+U)$ ground state) are available, and scales smoothly to cases where entire binary, ternary, or higher-dimensional chemical systems are calculated in $\mathrm{r}^{2} \mathrm{SCAN}$. We identified specific guidelines that can be used to target limited computational resources towards materials where $\mathrm{r}^{2} \mathrm{SCAN}$ calculations are likely to improve accuracy the most, and illustrated how the mixing scheme can be applied to solid and aqueous phase stability predictions.

\section{METHODS}

We employed the Vienna ab initio Simulation Package (VASP) $[50,51]$ v.6.1.1 in conjunction with v.54 of the projector-augmented wave (PAW) PBE pseudopotentials [52] for all $\mathrm{r}^{2} \mathrm{SCAN}$ calculations in this work. We employed a two-step high-throughput workflow described elsewhere [18], which comprises a structure optimization with PBEsol [25] to generate a initial guess of the charge density, followed by a subsequent structure optimization with $\mathrm{r}^{2}$ SCAN [24]. These calculations were force-converged, with a plane-wave energy cutoff of 680 $\mathrm{eV}$ and a bandgap-dependent $k$-point density [18, 53], which were developed to achieve a formation energy converged to within approximately $1 \mathrm{meV} /$ atom. We do not apply any energy corrections to the resulting $\mathrm{r}^{2} \mathrm{SCAN}$ energies. For context regarding corrections, in Appendix D we fit energy corrections to diatomic gases and show that the corrections that would be applied to $\mathrm{r}^{2} \mathrm{SCAN}$ are substantially smaller than those that have been widely used for $\operatorname{GGA}(+U)[14,15]$.

PBE calculations were retrieved from the Materials Project REST API [54]. Calculations for transition metal oxides and fluorides contained a Hubbard $U$ value and incorporated the GGA/GGA $+U$ mixing scheme of Jain et al. [9], in addition to empirical corrections applied to some chemical systems [10]. We refer to these calculations as $\mathrm{GGA}(+U)$ throughout this work.

\section{DATA AVAILABILITY}

All data referenced herein are publicly available in the Materials Project database [54]. At the time of this publication the database contains $\mathrm{r}^{2} \mathrm{SCAN}$ calcula- 
tions for approximately 33,000 materials, corresponding to $77 \%$ of all elements, binary, and ternary materials within $20 \mathrm{meV} /$ atom of the $\mathrm{GGA}(+U)$ convex energy hull. Our computational workflow has been implemented into the PYMATGEN [36] and ATOMATE [55] packages as of version 2020.1.28 and 0.9.5, respectively, for readers wishing to utilize it in their own work. The mixing scheme described herein is available in the MATERIALSPRojectDFTMixingSCHEME class in PYMATGEN [36] as of release 2022.1.20.

\section{ACKNOWLEDGEMENTS}

The authors gratefully acknowledge Stephan Lany (National Renewable Energy Laboratory) for contributing novel structures in the $\mathrm{Zn}-\mathrm{Sb}-\mathrm{N}$ system, Julia Yang (University of California, Berkeley) for contributing the $\mathrm{SnBr}_{2}$ structures, and Matthew McDermott (University of California, Berkeley) for helpful discussions regarding construction of phase diagrams with a shifted nitrogen chemical potential.

This work was intellectually led by the Materials Project, which is funded by the U.S. Department of Energy, Office of Science, Office of Basic Energy Sciences, Materials Sciences and Engineering Division, under Contract no. DE-AC02-05-CH11231: Materials Project program KC23MP. Additional support was also provided by the Data Infrastructure Building Blocks (DIBBS) Local Spectroscopy Data Infrastructure (LSDI) project funded by the National Science Foundation (NSF) under Award Number 1640899. A.S.R. acknowledges support via a Miller Research Fellowship from the Miller Institute for Basic Research in Science, University of California, Berkeley.

\section{AUTHOR CONTRIBUTIONS}

Ryan Kingsbury: Conceptualization, Software, Methodology, Data Curation, Formal Analysis, Visualization, Validation, Writing - Original Draft, Writing Review \& Editing. Andrew S. Rosen: Data Curation, Visualization, Formal Analysis, Validation, Writing Original Draft, Writing - Review \& Editing. Ayush Gupta: Formal Analysis, Visualization. Jason Munro: Software, Data Curation, Validation. Shyue Ping Ong: Methodology, Writing - Review \& Editing. Anubhav Jain: Methodology, Writing - Review \& Editing. Shyam Dwaraknath: Conceptualization, Methodology, Writing - Review \& Editing, Supervision. Matthew Horton: Conceptualization, Methodology, Writing - Review \& Editing, Supervision. Kristin Persson: Conceptualization, Methodology, Writing - Review \& Editing, Supervision, Funding Acquisition, Project Administration.

\section{COMPETING INTEREST STATEMENT}

The authors declare no competing financial interests.

\section{REFERENCES}

[1] A. Jain, S. P. Ong, G. Hautier, W. Chen, W. D. Richards, S. Dacek, S. Cholia, D. Gunter, D. Skinner, G. Ceder, and K. A. Persson, The Materials Project: A materials genome approach to accelerating materials innovation, APL Materials 1, 011002 (2013).

[2] S. Curtarolo, W. Setyawan, S. Wang, J. Xue, K. Yang, R. H. Taylor, L. J. Nelson, G. L. Hart, S. Sanvito, M. Buongiorno-Nardelli, N. Mingo, and O. Levy, Aflowlib.org: A distributed materials properties repository from high-throughput ab initio calculations, Computational Materials Science 58, 227 (2012).

[3] J. E. Saal, S. Kirklin, M. Aykol, B. Meredig, and C. Wolverton, Materials design and discovery with highthroughput density functional theory: The open quantum materials database (oqmd), JOM 65, 1501 (2013).

[4] S. Kirklin, J. E. Saal, B. Meredig, A. Thompson, J. W. Doak, M. Aykol, S. Rühl, and C. Wolverton, The Open Quantum Materials Database (OQMD): Assessing the accuracy of DFT formation energies, npj Computational Materials 1, 10.1038/npjcompumats.2015.10 (2015).

[5] K. Choudhary, K. F. Garrity, A. C. Reid, B. DeCost, A. J. Biacchi, A. R. H. Walker, Z. Trautt, J. HattrickSimpers, A. G. Kusne, A. Centrone, A. Davydov, J. Jiang, R. Pachter, G. Cheon, E. Reed, A. Agrawal,
X. Qian, V. Sharma, H. Zhuang, S. V. Kalinin, B. G. Sumpter, G. Pilania, P. Acar, S. Mandal, K. Haule, D. Vanderbilt, K. Rabe, and F. Tavazza, The joint automated repository for various integrated simulations (jarvis) for data-driven materials design, npj Computational Materials 6, 1 (2020).

[6] L. Himanen, A. Geurts, A. S. Foster, and P. Rinke, Datadriven materials science: Status, challenges, and perspectives, Advanced Science 6, 1900808 (2019).

[7] J. P. Perdew, K. Burke, and M. Ernzerhof, Generalized gradient approximation made simple, Phys. Rev. Lett. $\mathbf{7 7}, 3865$ (1996).

[8] D. C. Langreth and J. P. Perdew, Theory of nonuniform electronic systems. i. analysis of the gradient approximation and a generalization that works, Physical Review B 10.1103/PhysRevB.21.5469 (1980).

[9] A. Jain, G. Hautier, S. P. Ong, C. J. Moore, C. C. Fischer, K. A. Persson, and G. Ceder, Formation enthalpies by mixing GGA and GGA+U calculations, Physical Review B - Condensed Matter and Materials Physics 84, 1 (2011).

[10] A. Wang, R. Kingsbury, M. McDermott, M. Horton, A. Jain, S. P. Ong, S. Dwaraknath, and K. A. Persson, A framework for quantifying uncertainty in DFT energy 
corrections, Scientific Reports 11, 10.1038/s41598-02194550-5 (2021).

[11] C. Chen, Y. Zuo, W. Ye, X. Li, Z. Deng, and S. P. Ong, A critical review of machine learning of energy materials, Advanced Energy Materials 10, 1903242 (2020).

[12] P. Mori-Sánchez, A. J. Cohen, and W. Yang, Manyelectron self-interaction error in approximate density functionals, The Journal of chemical physics 125, 201102 (2006).

[13] J. P. Perdew, Climbing the ladder of density functional approximations, MRS Bulletin 38, 743 (2013).

[14] S. Grindy, B. Meredig, S. Kirklin, J. E. Saal, and C. Wolverton, Approaching chemical accuracy with density functional calculations: Diatomic energy corrections, Physical Review B - Condensed Matter and Materials Physics 87, 1 (2013).

[15] L. Wang, T. Maxisch, and G. Ceder, Oxidation energies of transition metal oxides within the GGA+U framework, Physical Review B - Condensed Matter and Materials Physics 73, 1 (2006).

[16] V. I. Anisimov, J. Zaanen, and O. K. Andersen, Band theory and mott insulators: Hubbard $\mathrm{u}$ instead of stoner i, Phys. Rev. B 44, 943 (1991).

[17] S. Grimme, A. Hansen, J. G. Brandenburg, and C. Bannwarth, Dispersion-Corrected Mean-Field Electronic Structure Methods, Chemical Reviews 116, 5105 (2016).

[18] R. Kingsbury, A. Gupta, C. Bartel, J. Munro, S. Dwaraknath, M. Horton, and K. Persson, Performance comparison of r2scan and scan metagga density functionals for solid materials via an automated, high-throughput computational workflow, ChemRxiv 10.33774/chemrxiv2021-gwm9m-v2 (2021).

[19] S. Lany, Semiconductor thermochemistry in density functional calculations, Physical Review B - Condensed Matter and Materials Physics 78, 1 (2008).

[20] M. Aykol and C. Wolverton, Local environment dependent GGA $+\mathrm{U}$ method for accurate thermochemistry of transition metal compounds, Physical Review B - Condensed Matter and Materials Physics 90, 1 (2014).

[21] R. Friedrich, D. Usanmaz, C. Oses, A. Supka, M. Fornari, M. Buongiorno Nardelli, C. Toher, and S. Curtarolo, Coordination corrected ab initio formation enthalpies, npj Computational Materials 5, 1 (2019), arXiv:arXiv:1811.08952v2.

[22] E. B. Isaacs and C. Wolverton, Performance of the strongly constrained and appropriately normed density functional for solid-state materials, Physical Review Materials 2, 1 (2018).

[23] G. Hautier, S. P. Ong, A. Jain, C. J. Moore, and G. Ceder, Accuracy of density functional theory in predicting formation energies of ternary oxides from binary oxides and its implication on phase stability, Phys. Rev. B 85, 155208 (2012).

[24] J. W. Furness, A. D. Kaplan, J. Ning, J. P. Perdew, and J. Sun, Accurate and numerically efficient r2scan meta-generalized gradient approximation, The Journal of Physical Chemistry Letters 11, 8208 (2020).

[25] J. P. Perdew, A. Ruzsinszky, G. I. Csonka, O. A. Vydrov, G. E. Scuseria, L. A. Constantin, X. Zhou, and K. Burke, Restoring the density-gradient expansion for exchange in solids and surfaces, Phys. Rev. Lett. 100, 136406 (2008).

[26] J. Sun, A. Ruzsinszky, and J. Perdew, Strongly Constrained and Appropriately Normed Semilocal Density
Functional, Physical Review Letters 115, 1 (2015).

[27] Y. Zhang, D. A. Kitchaev, J. Yang, T. Chen, S. T. Dacek, R. A. Sarmiento-Pérez, M. A. Marques, H. Peng, G. Ceder, J. P. Perdew, and J. Sun, Efficient first-principles prediction of solid stability: Towards chemical accuracy, npj Computational Materials 4, 10.1038/s41524-018-0065-z (2018).

[28] J. H. Yang, D. A. Kitchaev, and G. Ceder, Rationalizing accurate structure prediction in the meta-GGA SCAN functional, Physical Review B 100, 035132 (2019).

[29] W. Sun, A. Holder, B. Orvañanos, E. Arca, A. Zakutayev, S. Lany, and G. Ceder, Thermodynamic Routes to Novel Metastable Nitrogen-Rich Nitrides, Chemistry of Materials 29, 6936 (2017).

[30] J.-S. Park, Comparison study of exchange-correlation functionals on prediction of ground states and structural properties, Current Applied Physics 22, 61 (2021).

[31] C. J. Bartel, A. W. Weimer, S. Lany, C. B. Musgrave, and A. M. Holder, The role of decomposition reactions in assessing first-principles predictions of solid stability, npj Computational Materials 5, 10.1038/s41524-018-0143-2 (2019).

[32] D. Mejia-Rodriguez and S. B. Trickey, Deorbitalized meta-GGA exchange-correlation functionals in solids, Physical Review B 98, 10.1103/physrevb.98.115161 (2018).

[33] V. Stevanovic, S. Lany, X. Zhang, and A. Zunger, Correcting density functional theory for accurate predictions of compound enthalpies of formation: Fitted elementalphase reference energies, Physical Review B - Condensed Matter and Materials Physics 85, 1 (2012).

[34] See https://docs.materialsproject.org/methodology/ggaplus-u/.

[35] K. A. Persson, B. Waldwick, P. Lazic, and G. Ceder, Prediction of solid-aqueous equilibria: Scheme to combine first-principles calculations of solids with experimental aqueous states, Phys. Rev. B 85, 235438 (2012).

[36] S. P. Ong, W. D. Richards, A. Jain, G. Hautier, M. Kocher, S. Cholia, D. Gunter, V. L. Chevrier, K. A. Persson, and G. Ceder, Python Materials Genomics (pymatgen): A robust, open-source python library for materials analysis, Computational Materials Science 68, 314 (2013).

[37] T. R. Brumleve, Preparation, vapor pressure and thermochemistry of tin(II) bromide, ECS Proceedings Volumes 1992-16, 50 (1992).

[38] I. Levin, Nist inorganic crystal structure database (icsd) (2020).

[39] B. Patra, S. Jana, L. A. Constantin, and P. Samal, Correct structural phase stability of $\mathrm{FeS} 2, \mathrm{TiO} 2$, and $\mathrm{MnO} 2$ from a semilocal density functional, The Journal of Physical Chemistry C 125, 4284 (2021).

[40] W. Sun, C. J. Bartel, E. Arca, S. R. Bauers, B. Matthews, B. Orvananos, B.-R. Chen, M. F. Toney, L. T. Schelhas, W. Tumas, J. Tate, A. Zakutayev, S. Lany, A. M. Holder, and G. Ceder, A map of the inorganic ternary metal nitrides, Nature Materials 18, 732 (2019).

[41] E. Arca, J. D. Perkins, S. Lany, A. Mis, B. R. Chen, P. Dippo, J. L. Partridge, W. Sun, A. Holder, A. C. Tamboli, M. F. Toney, L. T. Schelhas, G. Ceder, W. Tumas, G. Teeter, and A. Zakutayev, Zn2sbn3: Growth and characterization of a metastable photoactive semiconductor, Materials Horizons 6, 1669 (2019). 
[42] W. Sun, S. T. Dacek, S. P. Ong, G. Hautier, A. Jain, W. D. Richards, A. C. Gamst, K. A. Persson, and G. Ceder, The thermodynamic scale of inorganic crystalline metastability, Science Advances 2, 10.1126/sciadv.1600225 (2016).

[43] A. L. Greenaway, C. L. Melamed, M. B. Tellekamp, R. Woods-Robinson, E. S. Toberer, J. R. Neilson, and A. C. Tamboli, Ternary nitride materials: Fundamentals and emerging device applications, Annual Review of Materials Research 51, 591 (2021).

[44] A. L. Greenaway, A. Loutris, K. N. Heinselman, C. L. Melamed, R. R. Schnepf, M. B. Tellekamp, R. Woods-Robinson, R. Sherbondy, D. J. Bardgett, S. R. Bauers, A. Zakutayev, S. T. Christensen, S. Lany, and A. Tamboli, Combinatorial synthesis of magnesium tin nitride semiconductors, Journal of the American Chemical Society 142, 8421 (2021), pMID: 32279492, https://doi.org/10.1021/jacs.0c02092.

[45] C. M. Caskey, R. M. Richards, D. S. Ginley, and A. Zakutayev, Thin film synthesis and properties of copper nitride, a metastable semiconductor, Mater. Horiz. 1, 424 (2014).

[46] C. M. Caskey, J. A. Seabold, V. Stevanović, M. Ma, W. A. Smith, D. S. Ginley, N. R. Neale, R. M. Richards, S. Lany, and A. Zakutayev, Semiconducting properties of spinel tin nitride and other IV3n4 polymorphs, Journal of Materials Chemistry C 3, 1389 (2015).

[47] A. Mis, S. Lany, G. L. Brennecka, and A. Tamboli, Exploring the phase space of zn2sbn3, a novel semiconducting nitride, Journal of Materials Chemistry C 9, 13904 (2021).

[48] A. M. Patel, J. K. Nørskov, K. A. Persson, and J. H. Montoya, Efficient pourbaix diagrams of many-element compounds, Physical Chemistry Chemical Physics 21, 25323 (2019).

[49] Z. Wang, X. Guo, J. Montoya, and J. K. Nørskov, Predicting aqueous stability of solid with computed pourbaix diagram using SCAN functional, npj Computational Materials 6, 10.1038/s41524-020-00430-3 (2020).

[50] G. Kresse and J. Furthmüller, Efficiency of ab-initio total energy calculations for metals and semiconductors using a plane-wave basis set, Computational Materials Science 6, 15 (1996).

[51] G. Kresse and J. Furthmüller, Efficient iterative schemes for ab initio total-energy calculations using a plane-wave basis set, Phys. Rev. B 54, 11169 (1996).

[52] P. E. Blöchl, Projector augmented-wave method, Phys. Rev. B 50, 17953 (1994).

[53] P. Wisesa, K. A. McGill, and T. Mueller, Efficient generation of generalized Monkhorst-Pack grids through the use of informatics, Physical Review B 93, 1 (2016).

[54] S. P. Ong, S. Cholia, A. Jain, M. Brafman, D. Gunter, G. Ceder, and K. A. Persson, The materials application programming interface (API): A simple, flexible and efficient API for materials data based on REpresentational state transfer (REST) principles, Computational Materials Science 97, 209 (2015).

[55] K. Mathew, J. H. Montoya, A. Faghaninia, S. Dwarakanath, M. Aykol, H. Tang, I. heng Chu, T. Smidt, B. Bocklund, M. Horton, J. Dagdelen, B. Wood, Z.-K. Liu, J. Neaton, S. P. Ong, K. Persson, and A. Jain, Atomate: A high-level interface to generate, execute, and analyze computational materials science workflows, Computational Materials Science 139, 140
(2017).

[56] L. Ward, A. Dunn, A. Faghaninia, N. E. Zimmermann, S. Bajaj, Q. Wang, J. Montoya, J. Chen, K. Bystrom, M. Dylla, K. Chard, M. Asta, K. A. Persson, G. J. Snyder, I. Foster, and A. Jain, Matminer: An open source toolkit for materials data mining, Computational Materials Science 152, 60 (2018). 


\section{APPENDICES}

\section{Appendix A: Comparing the relative stability of polymorphs}

The tendency of $\mathrm{r}^{2} \mathrm{SCAN}$ to stabilize unstable polymorphs is of particular interest. In this section, we assess the extent to which $\mathrm{r}^{2} \mathrm{SCAN}$ changes the energy above hull of polymorphs that are predicted to be unstable with $\mathrm{GGA}(+U)$, specifically focusing on structures with $0 \mathrm{meV} /$ atom $<\Delta E_{\text {hull }}^{\mathrm{GGA}(+U)}<50 \mathrm{meV} /$ atom. We define the parameter $\Delta \Delta E_{\text {hull }}^{\mathrm{GGA}(+U) \rightarrow \mathrm{r}^{2} \mathrm{SCAN}}$, which is the change in the energy above hull of the $\mathrm{GGA}(+U)$ polymorph when going from GGA $(+U)$ to $r^{2} \mathrm{SCAN}$. Thus, if the structure is predicted to be unstable by GGA $(+U)$ and moved closer to the hull with $\mathrm{r}^{2} \mathrm{SCAN}$, its $\Delta \Delta E_{\text {hull }}^{\mathrm{GGA}(+U) \rightarrow \mathrm{r}^{2} \mathrm{SCAN}}$ will be negative (and vice versa).

The results of this analysis are shown in Figure A.1. In the majority of cases, the change in $\Delta E_{\text {hull }}$ is small (within a few $\mathrm{meV} /$ atom). We note that for $95 \%$ of the unstable GGA $(+U)$ compounds within $50 \mathrm{meV} /$ atom of the $\mathrm{GGA}(+U)$ hull, $\Delta \Delta E_{\text {hull }}^{\mathrm{GGA}(+U) \rightarrow \mathrm{r}^{2} \mathrm{SCAN}}$ was greater than $-19 \mathrm{meV} /$ atom, and for $99 \%$ this value is $-36 \mathrm{meV} /$ atom. This means that compounds that are more than $19 \mathrm{meV} /$ atom above the GGA $(+U)$ hull are unlikely to appear on the $\mathrm{r}^{2} \mathrm{SCAN}$ hull, while compounds more than $36 \mathrm{meV} /$ atom above hull will only be stabilized by $\mathrm{r}^{2} \mathrm{SCAN}$ in especially rare circumstances.
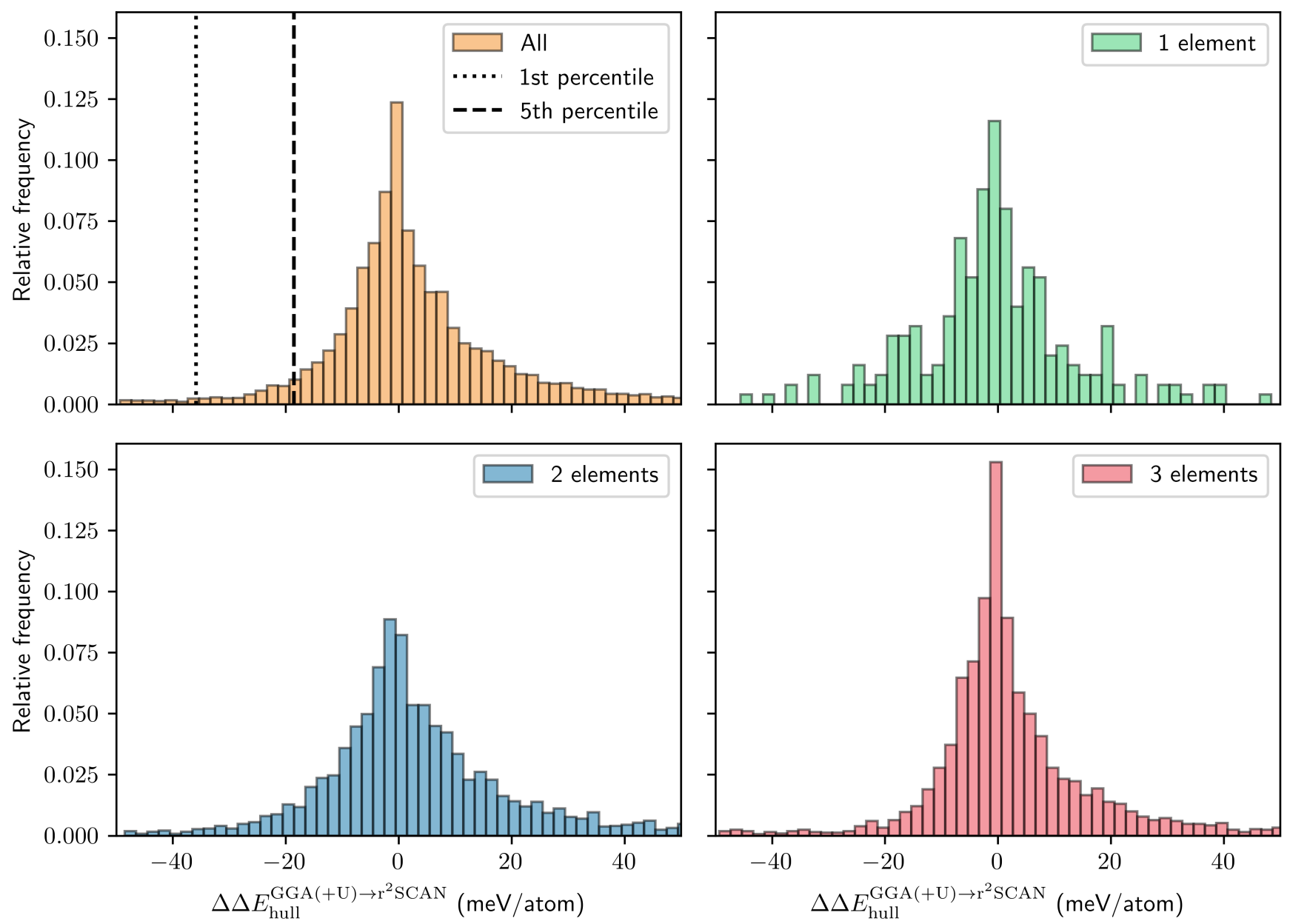

FIG. A.1. Change in energy above hull for polymorphs when going from GGA $(+U)$ to $\mathrm{r}^{2} \mathrm{SCAN}, \Delta \Delta E_{\text {hull }}^{\mathrm{GGA}(+U) \rightarrow \mathrm{r}^{2} \mathrm{SCAN}}$. Only polymorphs that are unstable in $\mathrm{GGA}(+U)$ and within $50 \mathrm{meV} /$ atom of the $\mathrm{GGA}(+U)$ hull are included. The data is broken down by the number of unique elements in the considered structures. 


\section{Appendix B: Differences in elemental ground-state structures and energies}

Here, we assess the frequency and magnitude with which $\mathrm{r}^{2} \mathrm{SCAN}$ changes the relative stability of elemental structures compared to GGA, which we define as the change in the energy above hull for a given structure when going from GGA to $\mathrm{r}^{2} \mathrm{SCAN}: \Delta \Delta E_{\text {hull }}^{\mathrm{GGA}} \rightarrow \mathrm{r}^{2} \mathrm{SCAN} \equiv \Delta E_{\text {hull }}^{\mathrm{r}^{2} \mathrm{SCAN}}-\Delta E_{\mathrm{hull}}^{\mathrm{GGA}}$. If $\mathrm{r}^{2} \mathrm{SCAN}$ yields the same elemental groundstate structure as GGA, then $\Delta \Delta E_{\text {hull }}^{\mathrm{GGA} \rightarrow \mathrm{r}^{2} \mathrm{SCAN}}=0$ by definition. If GGA predicts elemental structure A to be 2 $\mathrm{meV}$ /atom higher in energy compared to stable elemental structure B, but $\mathrm{r}^{2} \mathrm{SCAN}$ predicts elemental structure A to be stable and $2 \mathrm{meV} /$ atom lower in energy than elemental structure $\mathrm{B}$, then $\Delta \Delta E_{\text {hull }}^{\mathrm{GGA}} \rightarrow \mathrm{r}^{2} \mathrm{SCAN}=-4 \mathrm{meV} / \mathrm{atom}$. Note that because all structures considered in this section are elemental, no $U$ value is applied in this analysis.

Figure B.1 highlights the elements that have different ground-state structures between GGA and $\mathrm{r}^{2} \mathrm{SCAN}$ as well as the magnitude of this energy difference, where applicable. Based on the results in Figure B.1, even for the elements where $\mathrm{r}^{2} \mathrm{SCAN}$ stabilizes a different ground-state structure, the energy difference is often relatively small; the average value of $\Delta \Delta E_{\text {hull }}^{\mathrm{GGA} \rightarrow \mathrm{r}^{2} \mathrm{SCAN}}$ is $-30 \mathrm{meV} /$ atom (or $-12 \mathrm{meV} /$ atom if including the $0 \mathrm{meV} /$ atom change in energy above hull for the elements that do not have a different ground state). One pronounced exception is elemental Ce, for which the $\mathrm{r}^{2} \mathrm{SCAN}$ ground state structure is unstable in GGA by $2 \mathrm{meV} /$ atom, but the GGA ground state structure is unstable in $\mathrm{r}^{2} \mathrm{SCAN}$ by $223 \mathrm{meV} /$ atom. It should also be noted that, for some elements (i.e. Eu, Pu, Xe), $\mathrm{r}^{2} \mathrm{SCAN}$ does not a yield a DFT-optimized structure that matches the GGA ground-state structure (as determined by the PYMATGEN [36] StructureMatcher algorithm and subsequent manual inspection). This is likely due in part to an improved treatment of van der Waals interactions with $\mathrm{r}^{2} \mathrm{SCAN}$.

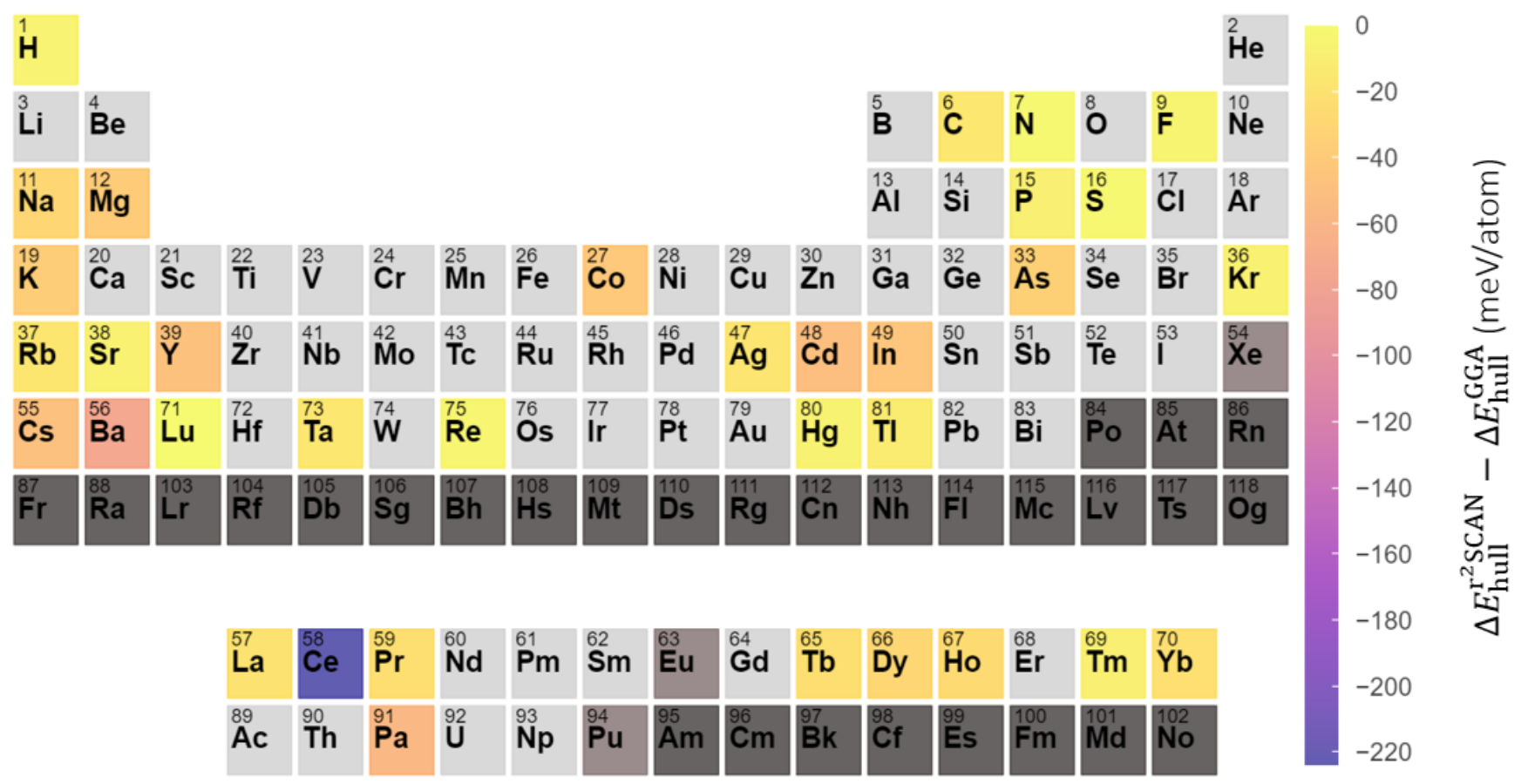

FIG. B.1. Energy differences in elemental ground-state structures computed with $\mathrm{r}^{2} \mathrm{SCAN}$ and GGA. The color bar indicates the amount by which the energy above hull of the unstable polymorph changed with $\mathrm{r}^{2} \mathrm{SCAN}$ compared to GGA. The elements shown as light gray have the same ground-state structure in both functionals. The elements shown as dark gray (i.e Po-Og)do not have entries on the Materials Project. The elements shown in brown (i.e. Xe, Eu, Pu) have have an $\mathrm{r}^{2} \mathrm{SCAN}$ ground state that that was different from the GGA ground state (and also different from all other GGA polymorphs). All elements were calculated without a $U$ value. 
Appendix C: Accuracy of $\mathbf{r}^{2}$ SCAN-edged ternary phase diagrams
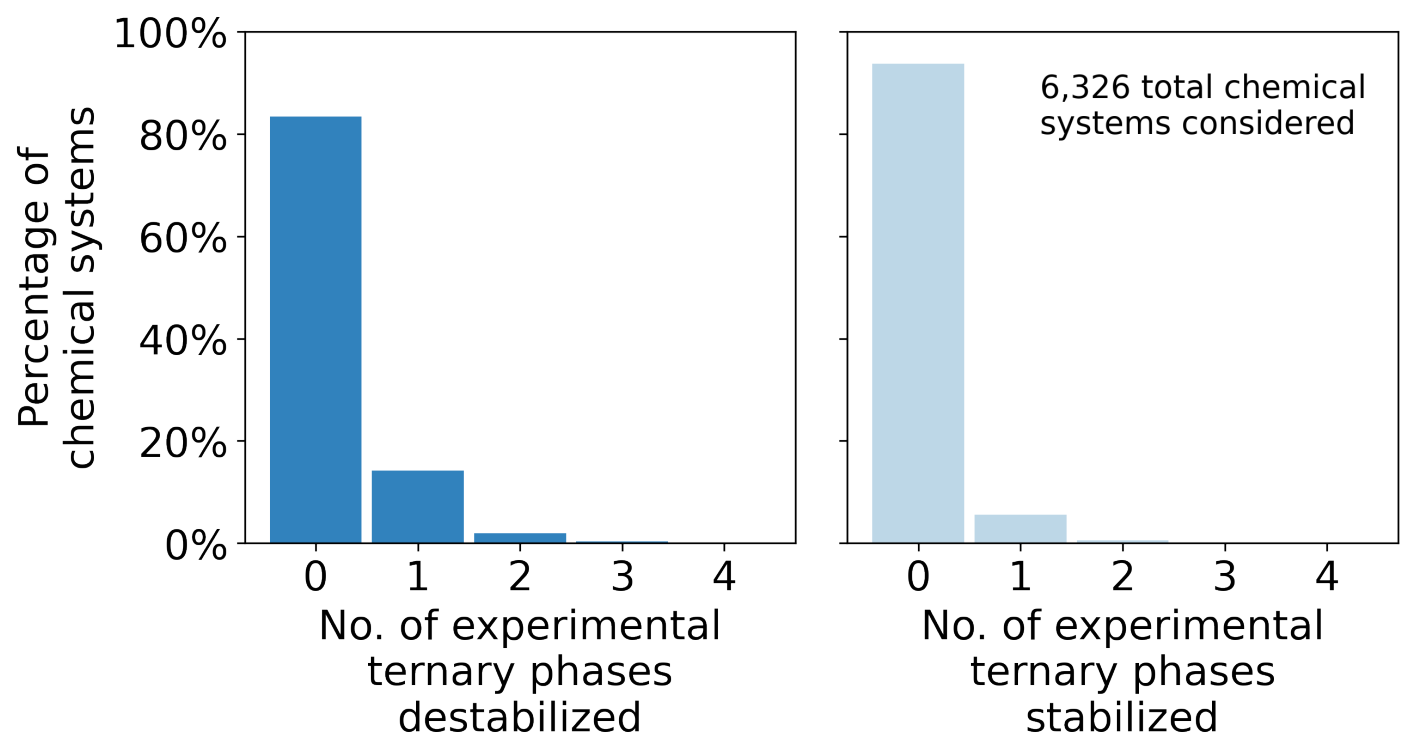

FIG. C.1. Changes to ternary phase stability predictions when constructing the hull using modified naive mixing as described in Section III A of the main text compared to pure GGA $(+U)$ for 6,326 ternary chemical systems. Left: number of experimental ternary phases predicted stable by pure GGA $(+U)$ that become unstable in the mixed diagram. Right: number of experimental ternary phases predicted unstable by pure GGA $(+U)$ that become stable in the mixed diagram. Experimental phases are phases that match one or more structures reported in the Inorganic Crystal Structure Database [38]. 


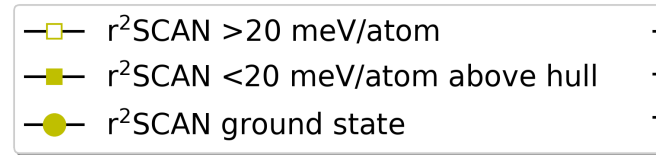

$r^{2}$ SCAN edges

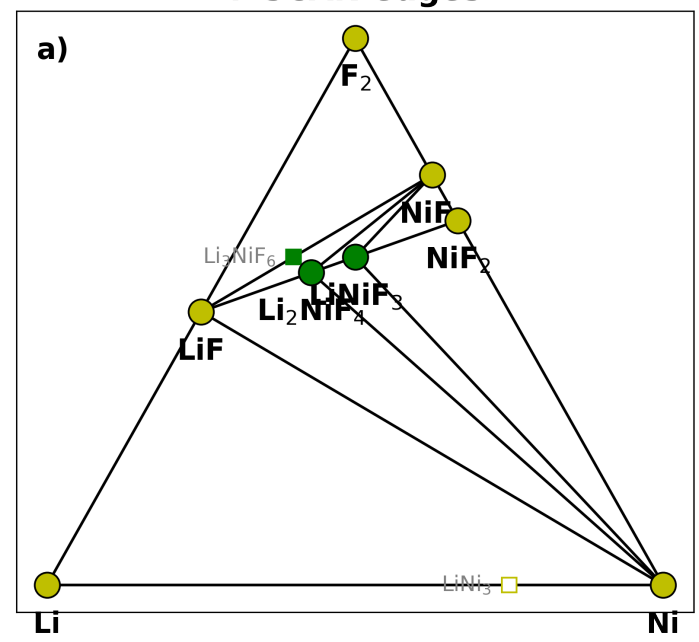

$-\square-G G A>20$ meV/atom above hull

$\rightarrow-\mathrm{GGA}<20 \mathrm{meV} / \mathrm{atom}$ above hull

-๑- GGA ground state

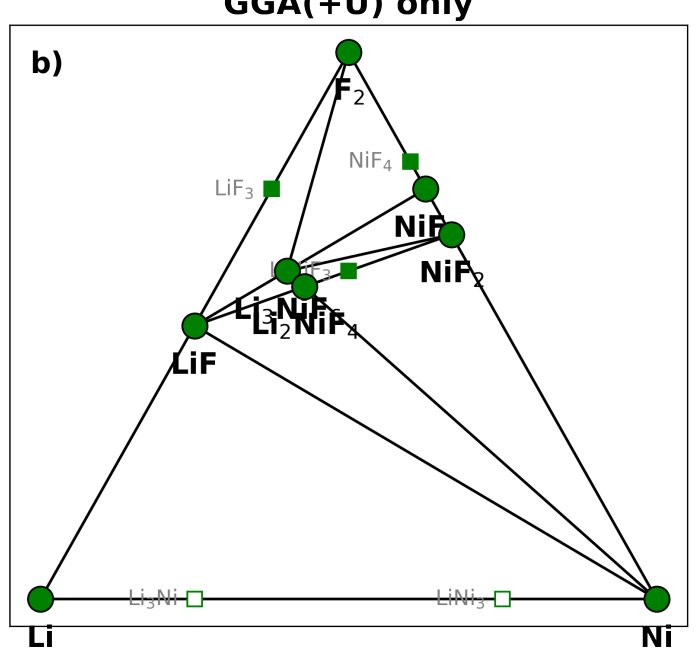

FIG. C.2. Example ternary phase diagram for the F-Li-Ni chemical system constructed by modified naive mixing as described in Section III A of the main text (left) and in pure GGA $(+U)$ (right). In the mixed diagram, the formation energies of all binary GGA $(+U)$ stable phases are replaced with $\mathrm{r}^{2}$ SCAN values. Some unstable binary GGA $(+U)$ phases are not shown. In the mixed diagram in this example, one stable ternary $\operatorname{GGA}(+U)$ phase is destabilized and one unstable ternary phase is stabilized. 


\section{Appendix D: Diatomic energy corrections}

One commonly used approach to addressing systematic errors in GGA functionals and improving the accuracy of DFT-predicted formation energies is to fit composition-dependent energy corrections to elements whose ground states are diatomic gases [14]. To evaluate the need for such corrections with meta-GGA calculations, we fit corrections to our $\mathrm{r}^{2} \mathrm{SCAN}$ energies following the same procedure and set of experimental compounds as Grindy et al [14]. $\mathrm{r}^{2} \mathrm{SCAN}$ and $\mathrm{GGA}(+U)$ energies were derived using the methods described in Section V, except that all energy corrections were removed from $\mathrm{GGA}(+U)$ energies prior to fitting. Experimental energies were obtained from the expt_formation_enthalpy_kingsbury dataset distributed with MATMINER [56], which is described further in Wang et al. and Kingsbury et al $[10,18]$.

The fitted corrections are summarized in Table D.1 and Figures D.1-D.5. Table D.1 also contains corrections fitted to $\operatorname{GGA}(+U)$ energies in this work and the corrections obtained by Grindy et al. [14] for comparison. $\mathrm{r}^{2} \mathrm{SCAN}$ corrections are substantially smaller than the corresponding $\mathrm{GGA}(+U)$ corrections in all cases. For example, the energy correction for $\mathrm{O}_{2}$ is $-0.351 \mathrm{eV} / \mathrm{O}_{2}$ for $\mathrm{r}^{2} \mathrm{SCAN}$, vs. $-1.286 \mathrm{eV} / \mathrm{O}_{2}$ when using $\mathrm{GGA}(+U)$ energies. The improvement in the accuracy of nitride formation energies is particularly notable (see Fig. D.2). Here, the correction is reduced from $0.811 \mathrm{eV} / \mathrm{N}_{2}$ with $\mathrm{GGA}(+U)$ energies to just $0.055 \mathrm{eV} / \mathrm{N}_{2}$ with $\mathrm{r}^{2} \mathrm{SCAN}$.

Given the small magnitude of the $\mathrm{r}^{2}$ SCAN energy corrections obtained here and considering that such corrections are inherently biased to simple chemistries by the nature of the fitting process, we elected not to apply any energy corrections and to use $\mathrm{r}^{2} \mathrm{SCAN}$ energies as-is when constructing phase diagrams. For detailed study of specific chemical systems (e.g. chlorides or oxides), modest improvements in the accuracy of formation energies might be obtained by applying the corrections shown here.

TABLE D.1. Diatomic energy corrections to $\mathrm{r}^{2} \mathrm{SCAN}$ formation energies $\left(\mathrm{eV} / \mathrm{X}_{2}\right.$ molecule)

\begin{tabular}{cccc}
\hline \hline Molecule & $\mathrm{r}^{2}$ SCAN & MP GGA $(+U)$ & Grindy et al. [14] \\
\hline $\mathrm{O}_{2}$ & -0.351 & -1.286 & -1.198 \\
$\mathrm{~N}_{2}$ & -0.055 & -0.811 & -0.892 \\
$\mathrm{H}_{2}$ & -0.017 & -0.312 & -0.284 \\
$\mathrm{~F}_{2}$ & -0.165 & -1.010 & -0.884 \\
$\mathrm{Cl}_{2}$ & -0.374 & -1.105 & -0.966 \\
\hline \hline
\end{tabular}




\section{Oxides}

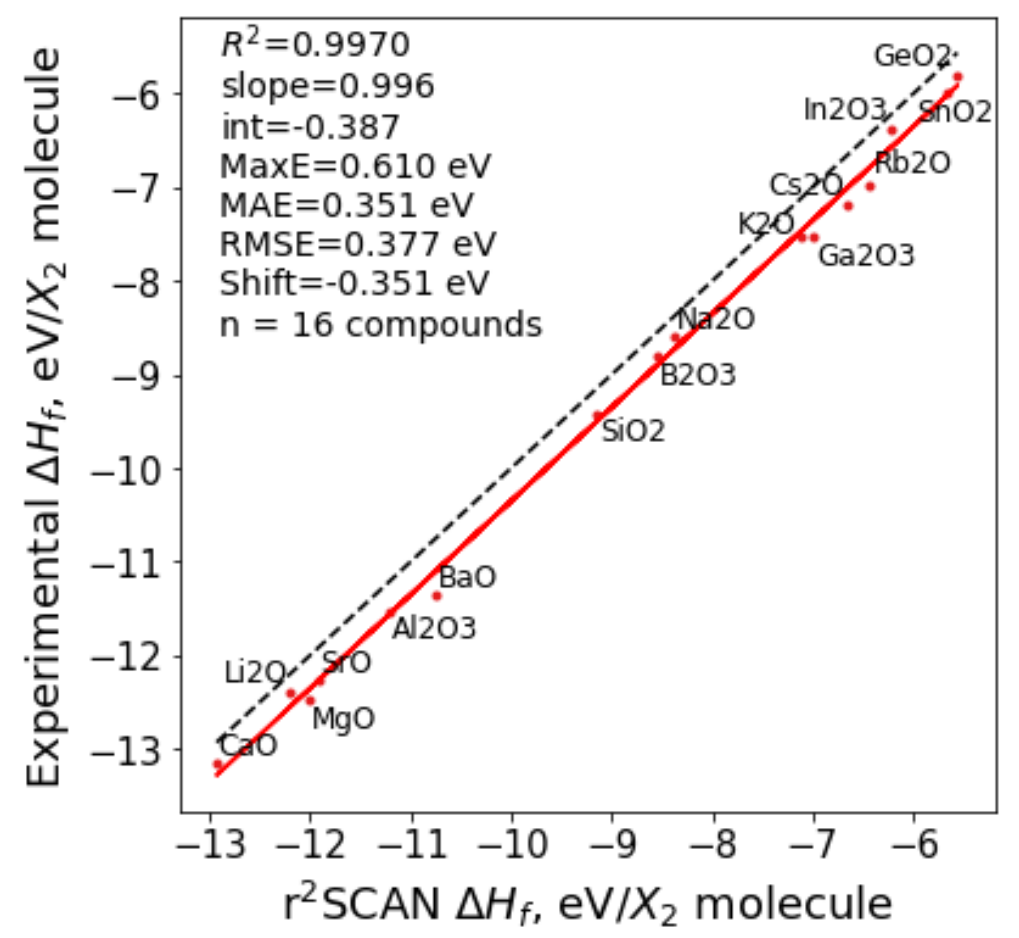

FIG. D.1. Experimental and $\mathrm{r}^{2} \mathrm{SCAN}-$ computed $\Delta H_{f}$ for oxide compounds. The compounds correspond to those selected by Grindy et al. [14]. 
Nitrides

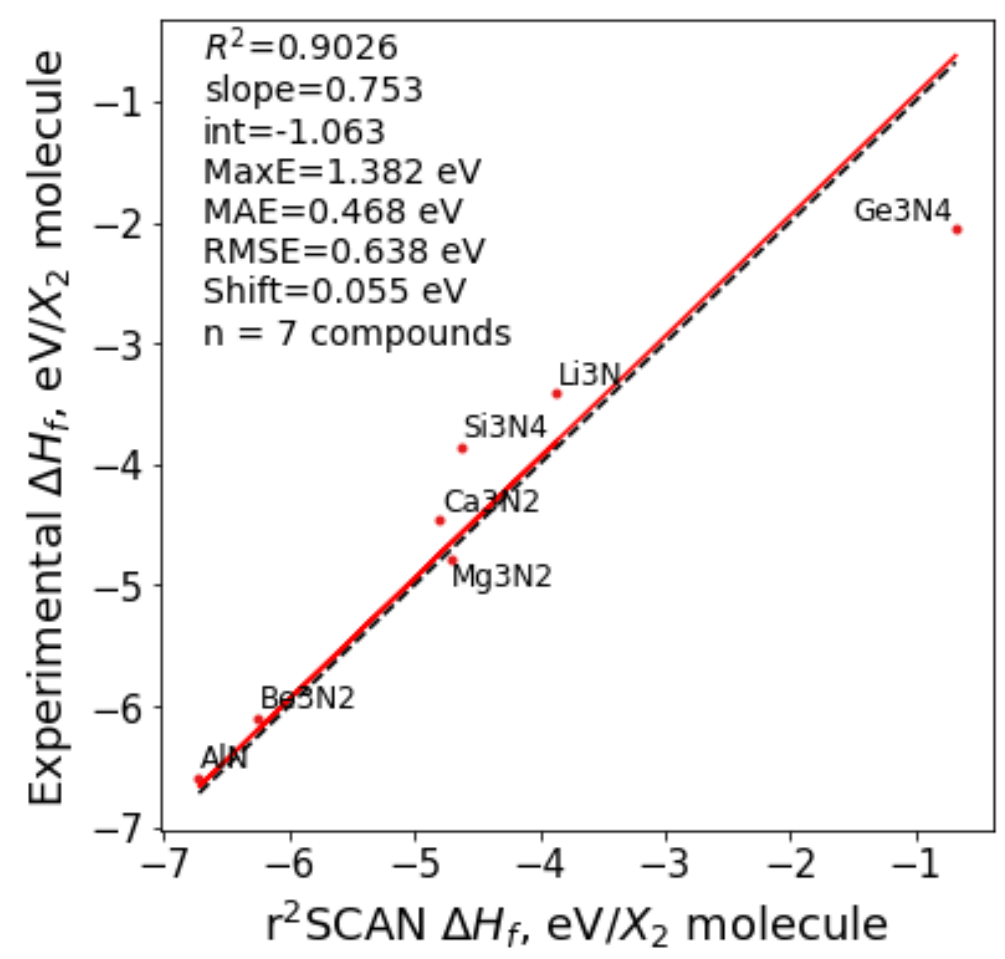

FIG. D.2. Experimental and $\mathrm{r}^{2} \mathrm{SCAN}-\mathrm{computed} \Delta H_{f}$ for nitride compounds. The compounds correspond to those selected by Grindy et al. [14]. 


\section{Chlorides}

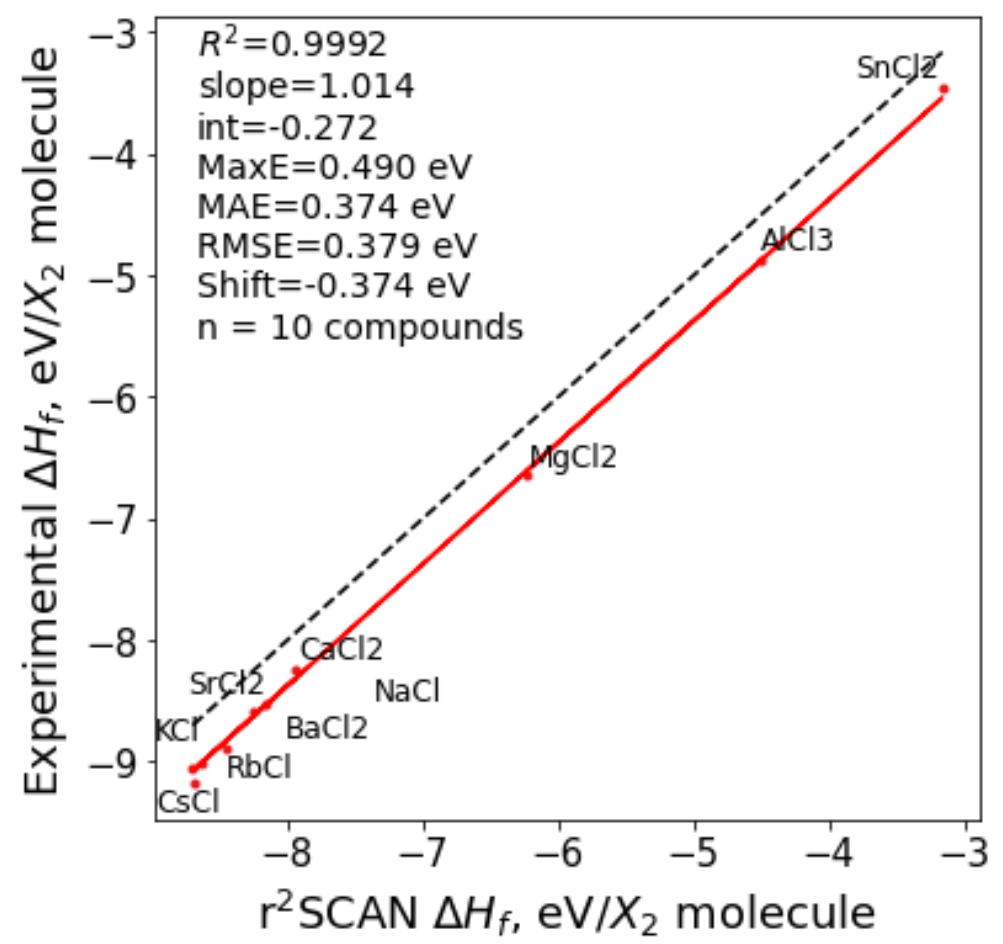

FIG. D.3. Experimental and $\mathrm{r}^{2} \mathrm{SCAN}-$ computed $\Delta H_{f}$ for chloride compounds. The compounds correspond to those selected by Grindy et al. [14]. 


\section{Hydrides}

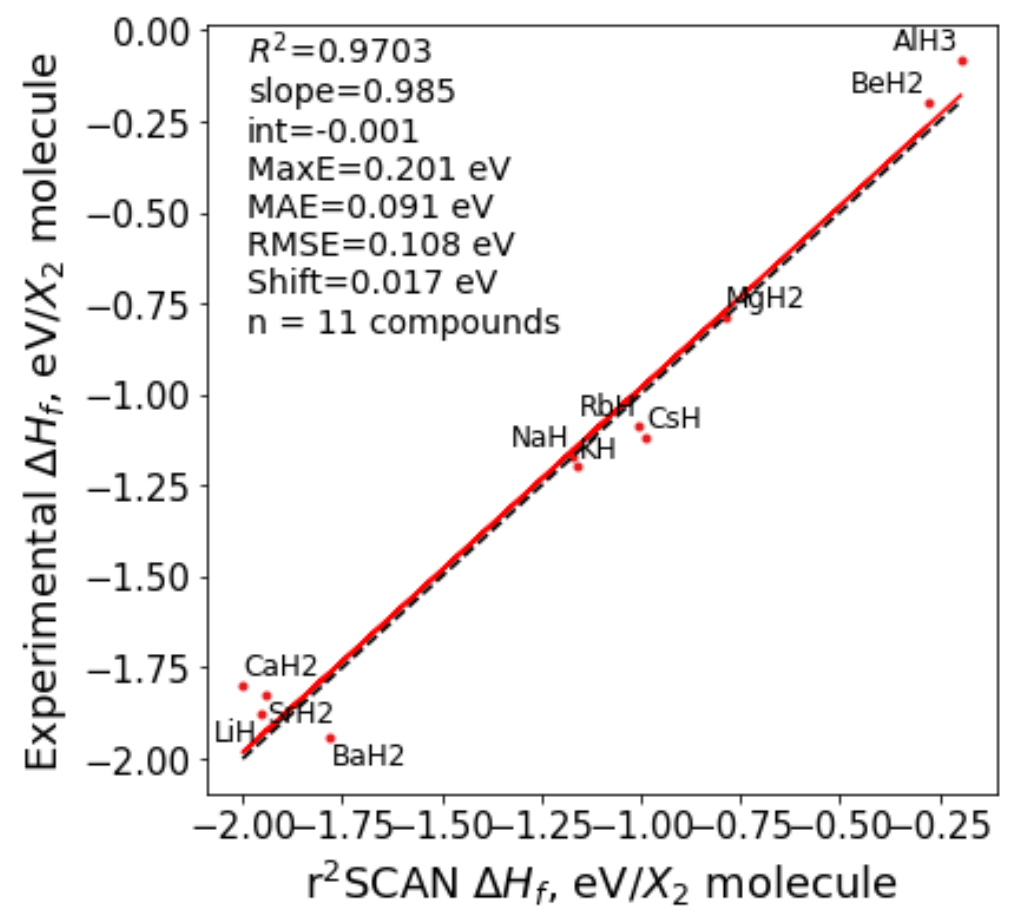

FIG. D.4. Experimental and $\mathrm{r}^{2} \mathrm{SCAN}$-computed $\Delta H_{f}$ for hydride compounds. The compounds correspond to those selected by Grindy et al. [14]. 
Fluorides

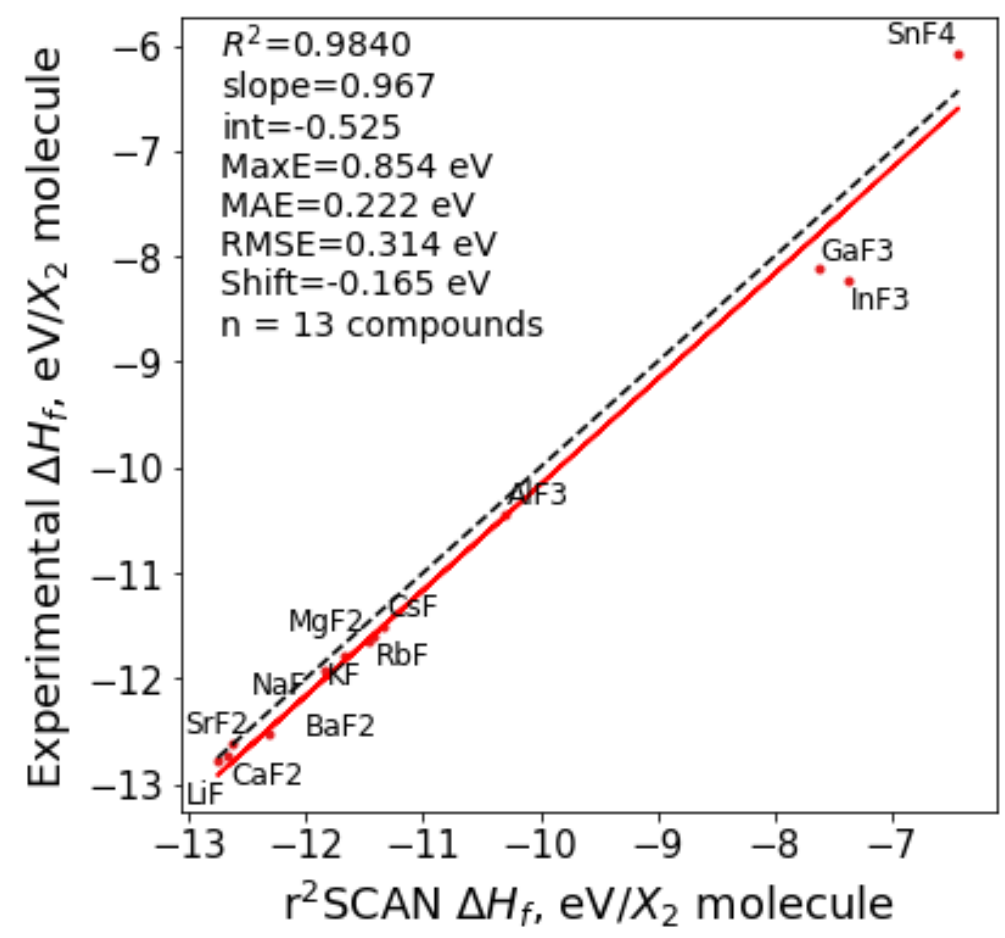

FIG. D.5. Experimental and $\mathrm{r}^{2} \mathrm{SCAN-computed} \Delta H_{f}$ for fluoride compounds. The compounds correspond to those selected by Grindy et al. [14]. 


\section{Appendix E: Description of tabulated data files}

Comma-separated value (.csv) files containing the composition, spacegroup, GGA $(+U)$ and $\mathrm{r}^{2} \mathrm{SCAN}$ energies of all materials used to construct the phase diagrams presented in the main text are included with the manuscript as Supporting Information. Each material (i.e., each distinct structure) is listed on a single row, with columns containing data from the corresponding $\mathrm{GGA}(+U)$ and/or $\mathrm{r}^{2} \mathrm{SCAN}$ calculations. Some columns may be blank if, for example, one of the calculations was not performed or the $\mathrm{r}^{2}$ SCAN-relaxed structure did not match the GGA $(+U)$-relaxed structure. The data are organized into the following columns:

- formula: The reduced formula of the material.

- spacegroup: The spacegroup of the material's structure.

- num_sites: The number of atoms in the material's structure.

- is_stable_1: Boolean value indicating whether the material is stable on the GGA $(+U)$ energy hull.

- entry_id_1: A unique identifier for the GGA $(+U)$ calculation, if one is present. The identifier comprises the material ID (MP-ID) of the material in the Materials Project database with the type of calculation (either "GGA(+U)" or "R2SCAN") appended.

- entry_id_2: A unique identifier for the $\mathrm{r}^{2} \mathrm{SCAN}$ calculation, if one is present. The identifier comprises the material ID (MP-ID) of the material in the Materials Project database with the type of calculation (either "GGA(+U)" or "R2SCAN") appended.

- run_type_1: The calculation type of entry_id_1 (always "GGA(+U)" in this work).

- run_type_2: The calculation type of entry_id_2 (always "R2SCAN" in this work).

- energy_1: The electronic energy (i.e., DFT energy) of the GGA $(+U)$ calculation, in eV/atom.

- energy_2: The electronic energy (i.e., DFT energy) of the $\mathrm{r}^{2} \mathrm{SCAN}$ calculation, in eV/atom.

- hull_energy_1: The energy of the GGA $(+U)$ convex hull at this material's composition, in eV/atom.

- hull_energy_2: The energy of the $\mathrm{r}^{2} \mathrm{SCAN}$ convex hull at this material's composition, in eV/atom. 